\title{
Counterfactual Thinking and Recency Effects in Causal Judgment
}

\author{
in press at Cognition
}

Paul Henne

Aleksandra Kulesza

Karla Perez

Augustana Houcek

\section{Author Note}

Paul Henne: https://orcid.org/0000-0002-3526-2911

Paul Henne is an Assistant Professor in the Department of Philosophy and is affiliated with the Neuroscience Program at Lake Forest College. Aleksandra Kulesza, Karla Perez, and Augustana Houcek are undergraduate researchers at Lake Forest College.

We have no conflicts of interest to disclose.

This research was funded by a startup grant from Lake Forest College to Paul Henne and the Reasons and Decisions Lab at Lake Forest College. Aleksandra Kulesza was supported by the Department of Philosophy at Lake Forest College. Karla Perez was supported by the Richter Scholarship at Lake Forest College. Augustana Houcek was supported by the James Rocco Data Research Scholarship at Lake Forest College. The authors thank Claire T. C. Henne, Louie T. D. Henne, Jennifer Jhun, Matthew Kelley, Josh Knobe, Nathan Liang, Janet McCracken, Kevin O’Neill, Roshni Patel, Matthew Stanley, and an audience in Felipe De Brigard's IMC Lab for comments on this project.

Correspondence should be addressed to Paul Henne 100 Durand Art Institute Lake Forest College 555 North Sheridan Road Lake Forest, IL 60045. phenne@lakeforest.edu. 
RECENCY EFFECTS IN CAUSAL JUDGMENT

\begin{abstract}
People tend to judge more recent events, relative to earlier ones, as the cause of some particular outcome. For instance, people are more inclined to judge that the last basket, rather than the first, caused the team to win the basketball game. This recency effect, however, reverses in cases of overdetermination: people judge that earlier events, rather than more recent ones, caused the outcome when the event is individually sufficient but not individually necessary for the outcome. In five experiments $(N=5507)$, we find evidence for the recency effect and the primacy effect for causal judgment. Traditionally, these effects have been a problem for counterfactual views of causal judgment. However, we argue that an extension of a recent counterfactual model of causal judgment explains both the recency and the primacy effect. In line with the predictions of our extended counterfactual model, we also find that, regardless of causal structure, people tend to imagine the counterfactual alternative to the more recent event rather than to the earlier one. Moreover, manipulating this tendency affects causal judgments in the ways predicted by this extended model: asking participants to imagine the counterfactual alternative to the earlier event weakens the interaction between recency and causal structure, and asking participants to imagine the counterfactual alternative to the more recent event strengthens the interaction between recency and causal structure. We discuss these results in relation to work on counterfactual thinking, causal modeling, and late-preemption. Keywords: causal judgment, recency, counterfactual thinking, imagination, late preemption, experimental philosophy
\end{abstract}


RECENCY EFFECTS IN CAUSAL JUDGMENT

$1 \quad$ Introduction

Louie and Claire would win a bet only if both make a three-point shot during a basketball game. Claire made a three-point shot right at the beginning of the game, and Louie made a three-point shot right at the buzzer. So, Louie and Claire won the bet. In this case, it seems more natural to claim that they won the bet because Louie made the three-point shot than it does to claim that they won the bet because Claire made the three-point shot. This difference in judgments is an example of the recency effect for causal judgment: in cases of joint-causation, people tend to judge that recent events are more causal than earlier ones (Lagnado \& Channon, 2008; Reuter et al., 2014; Spellman, 1997).

People's causal judgments, however, reverse in a slightly different causal structure. The recency effect occurs in cases of joint-causation, where each event is necessary and only jointly sufficient for the outcome. But consider a case of overdetermination — specifically a case of so-called late-preemptionwhere each event is individually sufficient but not individually necessary for the outcome. In this causal structure, Louie and Claire would win a bet if either makes a three-point shot during a basketball game. Claire made a three-point shot right at the beginning of the game, and Louie made a three-point shot right at the buzzer. So, Louie and Claire won the bet. In this case, it seems more natural to claim that they won the bet because Claire made the three-point shot than it does to claim that they won the bet because Louie made the three-point shot. This difference in judgments is an example of what we will call the primacy effect for causal judgment: in cases of overdetermination — specifically cases of late-preemption - people tend to judge that earlier events are more causal than recent ones (Chang, 2009; Lombrozo, 2010; Walsh \& Sloman, 2011).

Famously, one of the most prominent and influential accounts of causal judgment, counterfactual accounts — where causal judgments depend on counterfactual thinking — fails to explain the pervasive primacy effect and to give a unified explanation for these two effects (e.g., Gerstenberg et al., in press). In this article, we argue that an extension of a recent counterfactual model of causal judgment (Icard et al., 2017), however, can provide a unified explanation for the recency and primacy effects. The extension of this model predicts that people should tend to imagine the counterfactual alternatives to more recent 
RECENCY EFFECTS IN CAUSAL JUDGMENT

events when they show the recency and primacy effects and that manipulating this tendency should affect causal judgments. In this article, we test these predictions and find strong support for the extension of this model. Critically, these findings provide a counterfactual explanation for the primacy effect - i.e., judgments of late-preemption — a longstanding puzzle for counterfactual accounts of causal judgment, and they unify the explanation of the recency and primacy effects.

\subsection{A Counterfactual Account}

On counterfactual accounts of causal judgment, causal judgments depend on counterfactual thinking (Byrne, 2016; Gerstenberg et al., 2017; Lewis, 1974; Mackie, 1974). So, when people reason about whether some event caused an outcome, they do so by considering a counterfactual alternative to that event and asking whether the outcome would still occur in that alternative. Thus, in order to know if Louie making the three-point shot caused them to win the bet, people consider the situation where Louie did not make the three-point shot, and then they ask if Louie and Claire still would have won the bet. If Louie did not make the three-point shot at the buzzer, they would have lost the bet, so people should judge that Louie making the three-point shot made a difference-it caused them to win the bet.

In these scenarios, there are, however, many counterfactual alternatives in which the outcome would not occur (e.g., Hitchcock \& Knobe, 2009; Menzies, 2004; Phillips et al., 2015). For instance, Louie and Claire would not have won the bet if Claire had not made her shot at the beginning of the game, if they had not made the bet at all, or if basketball just never existed. Yet each of these counterfactual alternatives does not warrant a corresponding causal claim in the way that Louie's failing to make the buzzer shot does.

In order to account for this phenomenon, many counterfactual accounts of causal judgment do not treat all counterfactuals equally (Byrne, 2016; Bernstein, 2014; Halpern \& Hitchcock, 2014; Kahneman \& Miller, 1986; Phillips, et al., 2015). While researchers differ on exactly how to explain why people think some counterfactuals are relevant and others are not, one view suggests that it is a matter of how likely people are to simulate certain counterfactuals (Byrne, 2016; Icard et al., 2017; Kahneman \& Miller, 1986). On this kind of view, some factors affect people's propensity to simulate certain counterfactuals. 
RECENCY EFFECTS IN CAUSAL JUDGMENT

For instance, people are more likely to simulate the counterfactual to some abnormal action than the counterfactual to some normal action (Kahneman \& Miller, 1986; Icard et al., 2017; McCloy \& Byrne, 2000), and they are more likely to simulate the counterfactual to an action than the counterfactual to an inaction (Byrne \& McEleney, 2000; Henne et al., 2019; Kahneman \& Tversky, 1982). While it remains an open question as to why there are these features of counterfactual thinking at all, some argue that they aid goal-oriented decision making (e.g., Fazelpour, 2020).

This simulation view can be extended to recency effects in counterfactual thinking such that temporal order also affects people's tendency to simulate counterfactuals. Previous work on counterfactual thinking shows that people are more likely to think of counterfactual alternatives to more recent events than to earlier events (Miller \& Gunasegaram, 1990; Segura et al., 2002; Sherman \& McConnell, 1996; Walsh \& Byrne, 2004). In one study, researchers gave participants a passage in which Jones and Cooper will win some money if they each toss a coin and both coins land on the same side (Miller \& Gunasegaram, 1990). Jones tossed first and landed on heads, and Cooper tossed second and landed on tails. When asked to consider the possible ways in which they could have won the money, most participants judged that they would have won if only Cooper (the second player) tossed a head rather than if only Jones (the first player) tossed a tail (Miller \& Gunasegaram, 1990). This pattern of judgments also occurs when participants are asked to consider counterfactuals for more than two events in a temporal order (Byrne et al., 2000) and in studies involving 6- and 8-year-old children (Meehan \& Byrne, 2005). Researchers disagree as to why people consistently simulate counterfactuals to more recent events (Byrne, 2007; 2016), but some argue that this tendency occurs because particular contexts (Byrne et al., 2000; Walsh \& Byrne 2004) or working memory (Miller \& Gunasegaram, 1990) make these counterfactuals salient. Nonetheless, future work should further explore why people have this tendency at all.

Combining this work on counterfactual thinking and causal cognition, counterfactual accounts of causal judgment can provide a pretty straightforward explanation for the recency effect for causal judgment. People are more likely to simulate the counterfactual alternative to some recent event. Moreover, when the recent event is necessary for the outcome, the outcome does not occur in the 
RECENCY EFFECTS IN CAUSAL JUDGMENT

simulated counterfactual. This fact increases the extent to which people think that the recent event caused the outcome. In the case of Louie and Claire, each player's shot is necessary for them to win the bet. However, people are more inclined to imagine the counterfactual where Louie does not make the final shot than the counterfactual where Claire does not make her shot. And when people imagine this counterfactual, they imagine Louie and Claire losing the bet. As such, people are more inclined to think that Louie making the final shot made a difference: his shot caused them to win the bet.

While some counterfactual accounts can explain this recency effect for causal judgment, most have trouble with the primacy effect that occurs in cases of overdetermination. While there are distinct types of overdetermination discussed in the literature, we are focusing on judgments of what is called late preemption (Bernstein, 2016). In such cases, two events occur; each event is individually sufficient but not individually necessary for the outcome; if the first event failed to bring about the outcome, then the second would have brought it about; and the first event brings about the outcome (Paul \& Hall, 2013). Famously, counterfactual accounts of causation (Ganeri et al., 1996; Lewis, 1986; Lewis, 2000; Paul, 1998b; Paul \& Hall, 2013) and causal cognition (Gerstenberg et al., in press) have trouble explaining judgments of late preemption, which we are calling the primacy effect. In such cases, philosophers and non-philosophers accept that the earlier event caused the outcome (Gerstenberg et al., in press; Lombrozo, 2010; Paul \& Hall, 2013). Yet if people imagine the earlier event not occurring, the outcome still would have occurred in that scenario. So, the earlier event did not make a difference to the outcome, yet people still judge that the early event was the cause. For instance, if people imagine that Claire had not made her shot, Louie and Claire still would have won because Louie made his. But it still seems like Claire making her shot caused them to win the bet.

The extension of a recent counterfactual model of causal strength-i.e., the degree to which a factor is regarded as the cause of an outcome-however, shows promise for explaining and unifying the recency and primacy effects. This recent computational model, which we will refer to as the necessitysufficiency model (Icard et al., 2017), has been used to explain the effects of norms on causal judgments (Henne et al., 2021; Henne et al., 2017; Hitchcock \& Knobe, 2009; Kominsky et al., 2015; Morris et al., 
RECENCY EFFECTS IN CAUSAL JUDGMENT

2019; Samland et al., 2016; Willemsen \& Kirfel, 2018). Uniquely, this model's assumptions allow it to predict different patterns of causal judgments for different causal structures (see General Discussion for details). In joint-causation structures, it predicts that people will be more inclined to judge an event as causal when they are more inclined to simulate the counterfactual alternative to it, whereas in overdetermination cases people will be less inclined to judge the event as causal when they are more inclined to simulate the counterfactual alternative to it. The necessity-sufficiency model explains many effects produced by the abnormal-normal distinction's impact on causal judgment, and it has made some novel predictions that are confirmed in recent work (Icard et al., 2017; Kirfel \& Lagnado, 2019; Morris et al., 2019).

While some recent work has extended the necessity-sufficiency model to explain action-omission differences in causal judgments (Henne et al., 2019), we argue that a further, novel extension may explain both the recency and the primacy effects. If we extend the general approach of the necessity-sufficiency model to recency, then we need to introduce a new, critical assumption to it: people are more inclined to simulate counterfactuals to more recent events-regardless of causal structure. For instance, regardless of whether the causal structure is that of joint causation or overdetermination, people are more inclined to consider Louie not making his shot than they are to imagine Claire not making hers. ${ }^{1}$

With this new assumption on the extended necessity-sufficiency model, the model predicts that people judge the causal strength of recent or earlier events differently as a function of causal structure. In joint-causation cases, people are more inclined to judge the recent event as causal because they are more inclined to simulate the counterfactual alternative to it. For instance, people are more inclined to imagine Louie not making his shot, and in that situation, Louie and Claire do not win the bet. These simulations highlight that Louie's shot made a difference to the outcome. Hence, there is a predicted recency effect in causal judgment. In overdetermination cases, however, people are less inclined to judge the recent event

\footnotetext{
${ }^{1}$ Importantly, this assumption holds regardless of whether people make a causal judgment about the earlier or the more recent event. As such, it is not the demand of a causal judgment task that encourages people to simulate counterfactuals to more recent events.
} 
RECENCY EFFECTS IN CAUSAL JUDGMENT

as causal because they are more inclined to simulate the counterfactual alternative to it. ${ }^{2}$ For instance,

people are more inclined to imagine Louie not making his shot, and in that situation Louie and Claire still win the bet. These simulations highlight that Louie's shot did not make a difference to the outcome.

Hence, there is a predicted primacy effect in overdetermination structures. Thus, the extension of the necessity-sufficiency model provides a unified explanation for the recency and primacy effects for causal judgment. In doing this, it also provides a novel counterfactual explanation for judgments of latepreemption.

\subsection{The Present Studies}

In this article, we tested the predictions of our proposed extension of the necessity-sufficiency model. We investigated the recency and primacy effects for causal judgment and the interaction between causal structure and recency (i.e., temporal order) using continuous measures of causal strength (Experiments 1 and 2). We found an interaction between causal structure and recency reflecting the presence of both effects. We then investigated which event (earlier or later) people imagine happening differently (Experiment 2). In Experiment 2, participants reported imagining the counterfactual to the more recent event — regardless of causal structure. Using materials from Experiment 2, we found that manipulating this tendency affected causal judgments in some of the ways predicted by the model (Experiment 3): asking participants to imagine the counterfactual alternative to earlier events weakened the interaction between recency and causal structure, but asking participants to imagine the counterfactual alternative to the recent event only qualitatively strengthened the interaction between recency and causal structure.

In Experiments 1-3, participants' judgments about the statistical normality of the early or late event happening could have affected or explained our results (Supplemental Experiment 1). So, we then

\footnotetext{
${ }^{2}$ In overdetermination structures, even if one event did not happen, the outcome would still have happened. Some philosophers and cognitive scientists categorize these alternative possibilities, which we call counterfactuals, as semifactuals (Goodman, 1947; McCloy \& Byrne, 2002; Parkinson \& Byrne, 2017). Others disagree with this taxonomy of counterfactuals (Bennett, 2004). While we refer to these alternative possibilities as counterfactuals throughout, we do not take a stand on the appropriate taxonomy here. We, however, do note that previous work shows that people's consideration of semifactuals decreases the strength of causal judgments (McCloy \& Byrne, 2002; Parkinson \& Byrne, 2017).
} 
RECENCY EFFECTS IN CAUSAL JUDGMENT

attempted to replicate the recency and primacy effects in a range of new vignettes that controlled for and measured perceived statistical normality. In Experiment 4, we found robust support for these two effects and strong evidence that they arise independently of effects driven by perceived statistical normality. Using some of the new materials from Experiment 4, we then ran an improved version of Experiment 3 that avoided the confound of perceived statistical normality (Experiment 5). In Experiment 5, asking participants to imagine the counterfactual alternative to earlier events weakened the interaction between recency and causal structure, and asking participants to imagine the counterfactual alternative to the recent event strengthened the interaction between recency and causal structure. In sum, we found strong support for the extended necessity-sufficiency model and our counterfactual explanation for the recency and primacy effects.

\section{$2 \quad$ Experiment 1}

The aim of this experiment was to determine if there is an interaction between recency and causal structure such that a recency effect for causal judgment occurs in joint-causation structures and a primacy effect for causal judgment occurs in overdetermination structures. Specifically, we wanted to know (1) if in joint-causation conditions, participants would be more inclined to agree that events that happened later (i.e., more recent), relative to earlier ones, caused the outcome and (2) if in overdetermination conditions, participants would be more inclined to agree that events that happened earlier, relative to later ones (i.e., more recent), caused the outcome.

In this experiment, we manipulated recency (whether the event happened earlier or the event happened later) and causal structure (joint-causation or overdetermination), and we measured participants' agreement with a causal statement. While earlier work found the recency and primacy effects independently, these studies used a variety of different dependent measures, and none of them directly investigated an interaction between causal structure and recency. Our design allowed us to investigate this interaction and the presence of these two effects, and our continuous dependent measure allowed us to investigate the causal strength of each event. In our experiments, we used agreement with a causal explanation as the dependent measure, consistent with previous work on related topics (e.g., Henne 
RECENCY EFFECTS IN CAUSAL JUDGMENT

et al., 2019). That is, rather than asking participants if the events in the vignette caused the outcome, we asked if the outcome occurred because of the different events in the vignette. Importantly, people distinguish causal explanation and causation, but when they agree that something caused the outcome there is no evidence that there is a difference between causal judgments and causal explanations (Livengood \& Machery, 2010). We had no a priori hypothesis about the 'cause' and 'because' constructions of our dependent measure producing different results; we expected people to respond to a causal explanation or a causal statement similarly for recency effects. As such, we chose to use the more natural 'because' construction. If there are hypotheses for why people would respond differently to 'cause' and 'because' constructions, future work could examine these differences.

\subsection{Sample size}

A pilot study using only the joint-causation conditions found a recency effect $(p=.004)$ with an effect size of $d=.34$. We calculated the number of participants required for $d=.34$ for $\alpha=.05$ at .85 power. We required 156 participants for each condition. Expecting a 3-5\% drop-out rate, we aimed to recruit 650 participants.

\subsection{Participants}

We recruited all participants in all experiments in this manuscript on Prolific (www.prolific.co). All participants were United States nationals, were born in and resided in the United States, spoke English as their first language, had a 99\% approval rating on Prolific, and had not taken part in our pilot study. A total of 655 participants completed the experiment that was programmed in Qualtrics (www.qualtrics.com). 15 participants reported not paying attention, so they were excluded. We analyzed data from the remaining 640 participants $\left(M_{\text {age }}=32, S D=12.22\right.$, Range $_{\text {age }}=[18-75], 49 \%$ female $)$. After completing the survey, participants were compensated $\$ 0.27$.

\subsection{Materials and Procedure}

We advertised the experiment as "A Study About Your Judgments" and included the description: "Participants will be asked to read a short passage and then answer some questions about it." Participants 
RECENCY EFFECTS IN CAUSAL JUDGMENT

were told that they would be compensated $\$ 0.27$ upon completion of the study. Before participants entered the experiment, they read a consent form.

After they consented to participation, participants were randomly assigned to 1 of 4 conditions in a 2 (Recency: early, late) $\times 2$ (Structure: joint-causation, overdetermination) between-participants design . Each participant read a vignette and answered the causal question (Table 1). They were asked for their level of agreement with the causal statement on a $-50-50$ scale $[-50=$ strongly disagree, $0=$ neutral, $50=$ strongly agree]. Participants were then asked for basic demographic information and to respond to one explicit attention check that was used in all experiments in this manuscript (https://osf.io/42t36/).

All research in this manuscript was approved by the Human Subject Review Committee at Lake Forest College. All data collection was completed in all experiments in this manuscript prior to any analysis by the authors. All materials, data, and analysis code for all experiments in this manuscript are available at https://osf.io/74zg2/. The supplementary materials are available at https://osf.io/c95u3/. 
Joint-Causation, Early:

Louie is playing a game of basketball, and he made a bet with his

friends who are watching on the sidelines.

If Louie both makes a layup and makes a 3-point shot during the game, then he'll win $\$ 100$.

Just when the game started, Louie immediately got the ball at the 3point line. He looked to the basket, dribbled in, and then made a

layup right at the beginning of the game.

Louie and his friends continued playing, but as hard as he tried, Louie couldn't make another shot.

And then right at the end of the game as the clock was winding down, Louie got the ball at the 3-point line. He looked to the basket, focused his shot, and made a 3-point shot right at the buzzer. Then the game ended.

Because Louie would win $\$ 100$ if he both made a layup and a 3-point shot, Louie won $\$ 100$.

Overdetermination, Early:

Louie is playing a game of basketball, and he made a bet with his

friends who are watching on the sidelines.

If Louie either makes a layup or makes a 3-point shot during the game, then he'll win $\$ 100$.

Just when the game started, Louie immediately got the ball at the 3 point line. He looked to the basket, dribbled in, and then made a layup right at the beginning of the game.

Louie and his friends continued playing, but as hard as he tried, Louie couldn't make another shot.

And then right at the end of the game as the clock was winding down, Louie got the ball at the 3-point line. He looked to the basket, focused his shot, and made a 3-point shot right at the buzzer. Then the game ended.

Because Louie would win $\$ 100$ if he either made a layup or a 3-point shot, Louie won $\$ 100$.

Causal Question:

To what extent do you agree with the following statement about the passage you just read?

Louie won the $\$ 100$ bet because he made the layup.

Table 1. All vignettes used in Experiment 1 and the dependent variable.

\section{$2.4 \quad$ Results}

Critically, there was an interaction between causal structure and recency $\left(F(1,636)=109.67, p<.001, \eta^{2} p\right.$ $=.15, C I[.11, .19])$. There was no main effect of causal structure $\left(F(1,636)=2.62, p=.10, \eta^{2}{ }_{p}=.00, C I\right.$

$[.00, .02])$, but there was a main effect of recency $\left(F(1,636)=16.50, p<.001, \eta_{p}^{2}=.02, C I[.01, .05]\right)$.

Joint-Causation, Late:

Louie is playing a game of basketball, and he made a bet with his friends who are watching on the sidelines.

If Louie both makes a layup and makes a 3-point shot during the game, then he'll win $\$ 100$.

Just when the game started, Louie immediately got the ball at the 3point line. He looked to the basket, focused his shot, and made a 3point shot right at the beginning of the game.

Louie and his friends continued playing, but as hard as he tried, Louie couldn't make another shot.

And then right at the end of the game as the clock was winding down, Louie got the ball at the 3-point line. He looked to the basket, dribbled in, and then made a layup right at the buzzer. Then the game ended.

Because Louie would win $\$ 100$ if he both made a layup and a 3-point shot, Louie won $\$ 100$

Overdetermination, Late:

Louie is playing a game of basketball, and he made a bet with his friends who are watching on the sidelines.

If Louie either makes a layup or makes a 3-point shot during the game, then he'll win $\$ 100$.

Just when the game started, Louie immediately got the ball at the 3point line. He looked to the basket, focused his shot, and made a 3point shot right at the beginning of the game.

Louie and his friends continued playing, but as hard as he tried, Louie couldn't make another shot.

And then right at the end of the game as the clock was winding down, Louie got the ball at the 3-point line. He looked to the basket, dribbled in, and then made a layup right at the buzzer. Then the game ended.

Because Louie would win $\$ 100$ if he either made a layup or a 3-point shot, Louie won $\$ 100$ 
RECENCY EFFECTS IN CAUSAL JUDGMENT

To decompose the interaction between structure and recency, we computed tests of simple main effects. In line with the recency effect, participants in the joint-causation condition agreed that the recent event $(M=28.81, S D=27.96, n=159)$ was more causal than the earlier one $(M=11.71, S D=36.38, n=$ 162) $(t(319)=-4.71, p<.001, d=-.52, C I[-.75,-.30])$. In line with the primacy effect, participants in the overdetermination condition agreed that the earlier event $(M=35.33, S D=28.01, n=161)$ was more causal than the recent one $(M=-3.44, S D=40.85, n=158)(t(317)=9.90, p<.001, d=1.11, C I[.87$, 1.34]) (Figure 1).
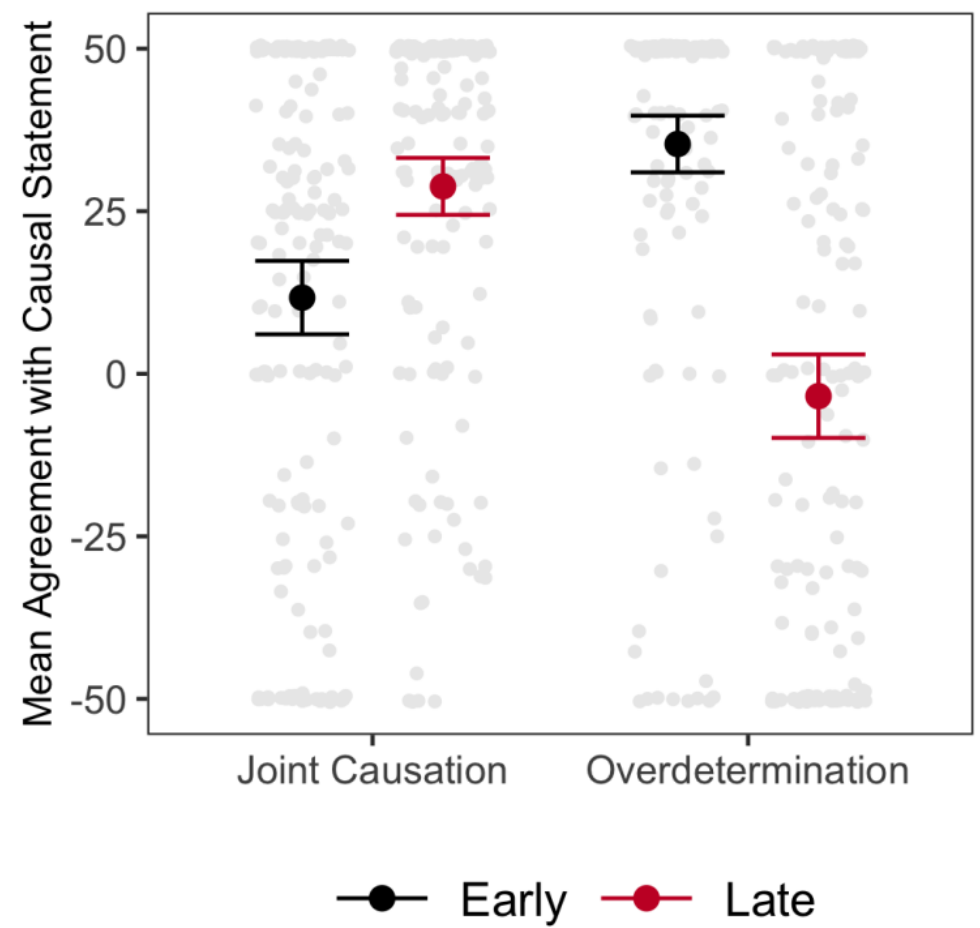

Figure 1. Mean agreement with the causal statement in Experiment 1. Error bars indicate 95\% confidence intervals. Light grey points represent individual participant responses evenly jittered.

\subsection{Discussion}

In Experiment 1, we found an interaction between recency and causal structure, reflecting the recency effect for causal judgment in joint-causation structures and a primacy effect for causal judgment in overdetermination structures. These results are consistent with previous work that finds a recency effect 
RECENCY EFFECTS IN CAUSAL JUDGMENT

in joint-causation structures (Lagnado \& Channon, 2008; Reuter et al., 2014; Spellman, 1997) and a primacy effect in overdetermination structures (Chang, 2009; Lombrozo, 2010; Mandel, 2003; Walsh \& Sloman, 2011). While previous work has used distinct stimuli and different measures to investigate these effects separately, our materials uniquely allowed us to investigate directly the interaction between causal structure and recency.

There is a worry about the generalizability of our findings in relation to previous work on this topic. In our vignette, only a single agent performed both actions that caused the outcome. Much of the earlier work on causal judgment found recency effects in joint-causation structures in situations where distinct agents performed the actions (e.g., Reuter et al., 2014). To ensure that participants saw the individual actions as distinct events, we revised our vignette in Experiment 2 so that it includes another agent performing a completely distinct action.

A second, more important concern is about the extent to which people consider the counterfactual alternatives to the events when they are making their causal judgments. The extension of the necessitysufficiency model assumes that people are—regardless of causal structures—more inclined to consider the counterfactual to the recent event and that this tendency produces the recency and primacy effects. In Experiment 1, we did not measure people's consideration of the counterfactual alternatives directly, so we cannot conclude that this was the case in Experiment 1.

Some work has investigated this tendency in people's counterfactual thinking. As we mentioned above, people tend to imagine the counterfactual alternatives to the more recent events in joint-causation structures (e.g., Miller \& Gunasegaram, 1990). However, some work has found that people consider the counterfactual alternatives to the earlier events rather than the most recent in overdetermination structures (Walsh \& Byrne, 2004; Mandel, 2003). Participants in one study read a passage where two players can win some money if one or the other, or both, pick a red card from a deck (Walsh \& Byrne, 2004: Experiment 3). The players, John and Michael, each drew a card. John drew first and picked a black card, and Michael drew second and picked a black card. So, they lost. Of the participants who imagined counterfactual alternatives for just one event (50\%), 33\% imagined the counterfactual alternative to the 
RECENCY EFFECTS IN CAUSAL JUDGMENT

earlier event and 17\% did so for the later one (Walsh \& Byrne, 2004). That is, participants were more inclined to consider counterfactuals for the earlier event instead of the more recent event in overdetermination structures. Notably, the researchers also found this pattern of results for exclusive disjunctions, where either one event or the other, but not both, would bring about the outcome (Walsh \& Byrne, 2004: Experiment 1). These findings suggest that the recency effect for counterfactual thinking that is typically found in joint-causation structures can be reversed in overdetermination structures (Walsh \& Byrne, 2004: Experiment 3). However, when these researchers manipulated the context, thus the salience of the counterfactual alternatives, they actually found a recency effect for counterfactual thinking in exclusive-disjunctive cases that was similar to the effect observed in joint-causation structures (Walsh \& Byrne, 2004: Experiment 2). These findings suggest that while there is a recency effect for counterfactual thinking, this tendency can be manipulated by context, causal structure, and other counterfactual interventions that make certain counterfactual alternatives salient.

The extension of the necessity-sufficiency model's hypothesis is that when people display the recency and primacy effects for causal judgment, they are more inclined to imagine the counterfactual to the recent event-regardless of causal structure. We did not know, however, if participants in Experiment 1 were more inclined to imagine the counterfactual to the recent event or the earlier one. Because this tendency can change depending on the salience of the counterfactual alternatives to each event (Walsh \& Byrne, 2004), we needed to test directly which counterfactuals people tended to imagine in our scenarios. We do so in Experiment 2. If people were overall more likely to report that they imagined the counterfactual to the recent event, then we would be justified in attempting to manipulate this tendency.

\section{$3 \quad$ Experiment 2}

There were two aims of this experiment. First, we wanted to conceptually replicate the results from Experiment 1 when multiple agents, as opposed to just one, act to bring about an outcome. Just as in Experiment 1, we manipulated recency and causal structure, and we measured participants' agreement with a causal statement. Second, we investigated which event people imagined happening differently (early or late). Previous work on imagining counterfactual alternatives prompted participants with 
RECENCY EFFECTS IN CAUSAL JUDGMENT

something like the following: "John and Michael could have each won some money if only one of them had picked a different card, for instance if..." and participants wrote in their responses (Walsh \& Byrne, 2004). With this prompt, participants who identified one event could have identified what made a difference to the outcome rather than what they were just imagining happening differently. Because of our interest in the relationship between counterfactual thinking and causal judgments, we wanted to avoid using a prompt that potentially emphasized what made a difference to the outcome. To avoid this potential confound, we asked participants, "Think about what could have been different in the story that you just read. Which event do you imagine happening differently?" and we had them select either the early or the late event. We asked this question in fixed order before the causal question so that responses to the causal question did not affect people's responses to the counterfactual question. In line with our proposed extension of the necessity-sufficiency model, we predicted that - if we replicated the recency and primacy effects for causal judgment - people would be more likely overall to report that they imagined the counterfactual to the more recent event, rather than to the earlier one, regardless of causal structure.

\subsection{Sample size}

We aimed to recruit 650 participants just as we did in Experiment 1.

\subsection{Participants}

All participants were United States nationals, were born in and resided in the United States, spoke English as their first language, had a 99\% approval rating on Prolific, and had not taken part in our pilot study or in Experiment 1. A total of 650 participants completed the experiment that was programmed in Qualtrics. 15 participants reported not paying attention, so they were excluded. We analyzed data from the remaining 635 participants $\left(M_{\text {age }}=34, S D=12.97\right.$, Range $_{\text {age }}=[18-84], 51 \%$ female $)$. After completing the survey, participants were compensated $\$ 0.27$.

\subsection{Materials and procedure}

We advertised the experiment and compensated participants in the exact same way as we did in Experiment 1. Before participants entered the experiment, they read a consent form. 
RECENCY EFFECTS IN CAUSAL JUDGMENT

After they consented to participation, participants were randomly assigned to 1 of 4 conditions in a 2 (Recency: early, late) $\times 2$ (Structure: joint-causation, overdetermination) between-participants design. Each participant read a vignette and answered the counterfactual question and then the causal question (Table 2). For the counterfactual question, we randomized the order in which the events were presented between participants, and participants selected whether they imagined the early or the late event happening differently. The causal question was presented in the exact same way as in Experiment 1. Participants were then asked for basic demographic information and to respond to an attention check.

\section{$3.4 \quad$ Coding}

We coded participants' responses to the counterfactual questions for the planned analyses. We coded participants' responses in the early conditions such that those who selected "Louie making the 3-point shot right at the beginning of the game" were coded as "Early" and those who selected "Claire making the 3-point shot right at the buzzer" were coded as "Late." We coded participants' responses in the late conditions such that those who selected "Claire making the 3-point shot right at the beginning of the game" were coded as "Early" and those who selected "Louie making the 3-point shot right at the buzzer" were coded as "Late." 
RECENCY EFFECTS IN CAUSAL JUDGMENT

Joint-Causation, Early:

Louie and Claire are playing a game of basketball, and they made a

bet with their friends who are watching on the sidelines.

If both Louie makes a 3-point shot and Claire makes a 3-point shot during the game, then they'll win $\$ 100$ and split it down the middle.

Just when the game started, Louie immediately got the ball at the 3point line. He looked to the basket, focused his shot, and made a 3point shot right at the beginning of the game.

Louie, Claire, and their friends continued playing, but as hard as they tried, Louie and Claire couldn't make another shot.

And then right at the end of the game as the clock was winding down, Claire got the ball at the 3-point line. She looked to the basket, focused her shot, and made a 3-point shot right at the buzzer. Then the game ended.

Because they would win $\$ 100$ if both Louie made a 3-point shot and Claire made a 3-point shot, Louie and Claire won $\$ 100$ and split it down the middle.

\section{Overdetermination, Early:}

Louie and Claire are playing a game of basketball, and they made a bet with their friends who are watching on the sidelines.

If either Louie makes a 3-point shot or Claire makes a 3-point shot during the game, then they'll win $\$ 100$ and split it down the middle.

Just when the game started, Louie immediately got the ball at the 3 point line. He looked to the basket, focused his shot, and made a 3point shot right at the beginning of the game.

Louie, Claire, and their friends continued playing, but as hard as they tried, Louie and Claire couldn't make another shot.

And then right at the end of the game as the clock was winding down, Claire got the ball at the 3-point line. She looked to the basket, focused her shot, and made a 3-point shot right at the buzzer. Then the game ended.

Because they would win $\$ 100$ if either Louie made a 3-point shot or Claire made a 3-point shot, Louie and Claire won $\$ 100$ and split it down the middle.

Counterfactual Question, Early:

Think about what could have been different in the story that you just read. Which event do you imagine happening differently:

Louie making the 3-point shot right at the beginning of the game

Claire making the 3-point shot right at the buzzer

Causal Question:

To what extent do you agree with the following statement about the passage you just read?

Louie and Claire won the $\$ 100$ bet because Louie made the 3-point shot.
Joint-Causation, Late:

Louie and Claire are playing a game of basketball, and they made a bet with their friends who are watching on the sidelines.

If both Louie makes a 3-point shot and Claire makes a 3-point shot during the game, then they'll win $\$ 100$ and split it down the middle.

Just when the game started, Claire immediately got the ball at the 3point line. She looked to the basket, focused her shot, and made a 3point shot right at the beginning of the game.

Louie, Claire, and their friends continued playing, but as hard as they tried, Louie and Claire couldn't make another shot.

And then right at the end of the game as the clock was winding down, Louie got the ball at the 3-point line. He looked to the basket, focused his shot, and made a 3-point shot right at the buzzer. Then the game ended.

Because they would win $\$ 100$ if both Louie made a 3-point shot and Claire made a 3-point shot, Louie and Claire won $\$ 100$ and split it down the middle.

\section{Overdetermination, Late:}

Louie and Claire are playing a game of basketball, and they made a bet with their friends who are watching on the sidelines.

If either Louie makes a 3-point shot or Claire makes a 3-point shot during the game, then they'll win $\$ 100$ and split it down the middle.

Just when the game started, Claire immediately got the ball at the 3point line. She looked to the basket, focused her shot, and made a 3 point shot right at the beginning of the game.

Louie, Claire, and their friends continued playing, but as hard as they tried, Louie and Claire couldn't make another shot.

And then right at the end of the game as the clock was winding down, Louie got the ball at the 3-point line. He looked to the basket, focused his shot, and made a 3-point shot right at the buzzer. Then the game ended.

Because they would win $\$ 100$ if either Louie made a 3-point shot or Claire made a 3-point shot, Louie and Claire won $\$ 100$ and split it down the middle.

Counterfactual Question, Late:

Think about what could have been different in the story that you just read. Which event do you imagine happening differently:

Claire making the 3-point shot right at the beginning of the game

Louie making the 3-point shot right at the buzzer

Table 2. All vignettes used in Experiment 2 and the dependent variables. 


\section{RECENCY EFFECTS IN CAUSAL JUDGMENT}

\subsection{Results}

First, we investigated participants' responses to the counterfactual question. Participants were overall much more likely to select the late event $(68.50 \%)$ than the early one $(31.49 \%)$ as the one that they imagined happening differently $\left(\chi^{2}(1, N=635)=86.96, p<.001, O R=4.24, C I[3.13,5.74]\right)$. This tendency occurred both in the joint-causation $\left(\chi^{2}(1, N=317)=71.92, p<.001, O R=7.13, C I[4.53\right.$, 11.23]) and in the overdetermination conditions $\left(\chi^{2}(1, N=318)=22.18, p<.001, O R=2.70, C I[1.78\right.$, 4.08]). A binomial logistic regression showed that there was no interaction between recency and structure for participants' selection in the counterfactual question $(O R=1.02, C I[.51,2.01], p=.94)$ and no effect of recency $(O R=1.02, C I[.62,1.69], p=.84)$. However, there was a small main effect of structure $(O R=$ $.60, C I[.37, .96], p=.003)$ such that participants in the overdetermination conditions selected the early event more frequently than participants in the joint-causation condition (Figure 2B). Pairwise comparisons revealed that there were no significant differences between any of the individual conditions (all $p \mathrm{~s}>$.10) (see Supplemental Materials for exploratory analyses and discussion).

For the causal question, there was an interaction between causal structure and recency $(F(1,631)$ $\left.=74.71, p<.001, \eta_{p}^{2}=.11, C I[.07, .14]\right)$. There was also a main effect of causal structure $(F(1,631)=$ $\left.14.82, p<.001, \eta_{p}^{2}=.02, C I[.01, .05]\right)$ and recency $\left(F(1,631)=12.35, p<.001, \eta^{2}=.11, C I[.01, .04]\right)$.

To decompose the interaction effect between structure and recency, we computed tests of simple main effects. In line with the recency effect, participants in the joint-causation condition agreed that the recent event $(M=15.82, S D=30.22, n=158)$ was more causal than the earlier one $(M=2.32, S D=$ $33.17, n=159)(t(315)=-3.78, p<.001, d=-.42, C I[-.64,-.20])$. In line with the primacy effect, participants in the overdetermination condition agreed that the earlier event $(M=14.89, S D=31.91, n=$ 158) was more causal than the recent one $(M=-16.81, S D=36.14, n=160)(t(316)=8.28, p<.001, d=$ .93, $C I[.70,1.15])$ (Figure 2A). 

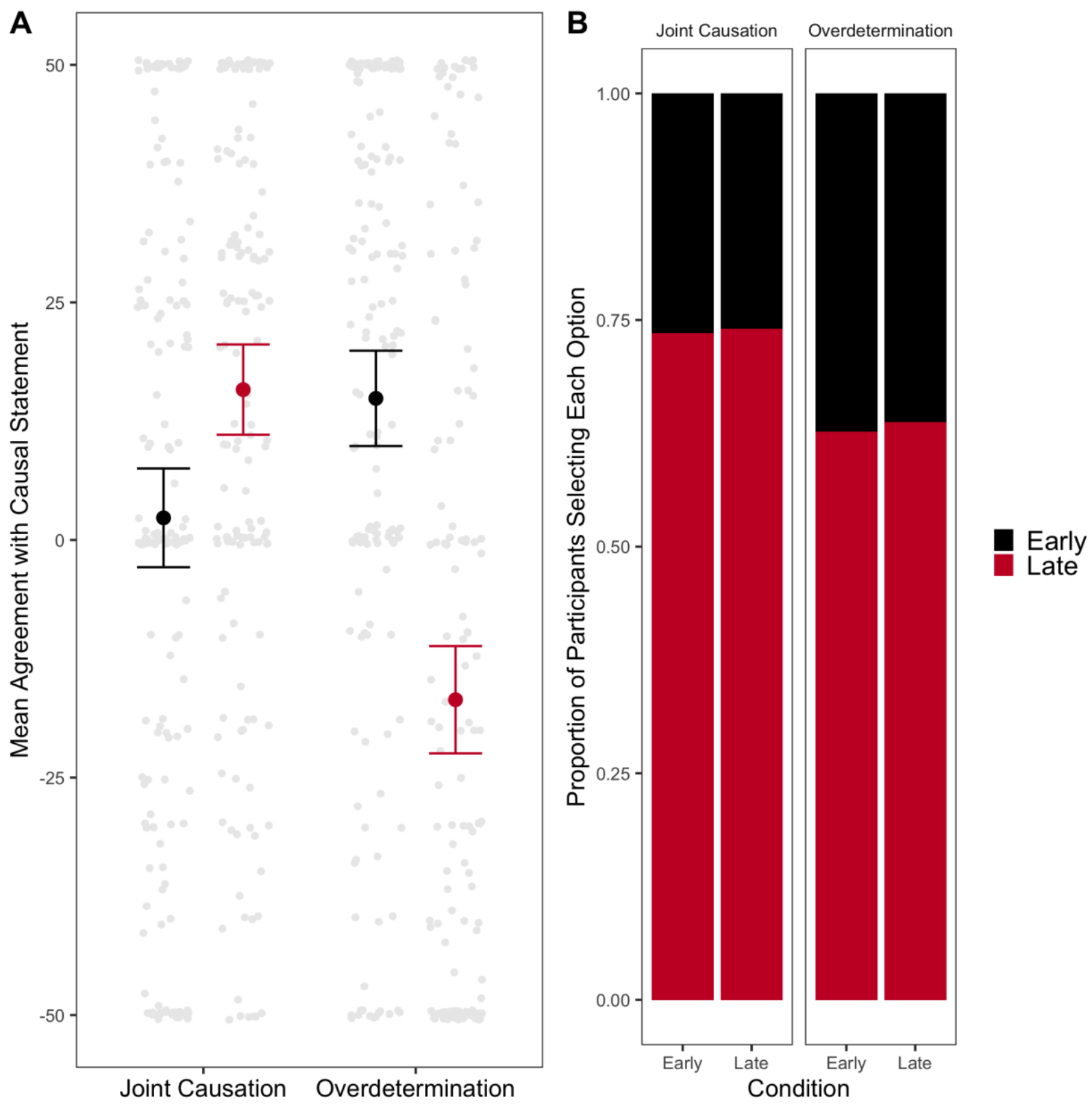

Figure 2. (A) Mean agreement with the causal statement in Experiment 2. Error bars indicate 95\% confidence intervals. Light grey points represent individual participant responses evenly jittered. $(B)$ Proportion of participants selecting each option for the counterfactual question as a function of structure and recency. 
RECENCY EFFECTS IN CAUSAL JUDGMENT

\subsection{Discussion}

In Experiment 2, we replicated the results of Experiment 1 in a situation where multiple agents, as opposed to just one, act to bring about the outcome; we found an interaction between recency and causal structure, reflecting the recency and primacy effects for causal judgment. These results confirm the reliability of the effects found in Experiment 1.

We also found that regardless of causal structure, participants overall tended to report that they imagined the counterfactual alternative to the more recent event. This finding was predicted by our proposed extension of the necessity-sufficiency model: when participants show the recency and primacy effect for causal judgment, they should also show a recency effect for counterfactual thinking in both joint-causation and overdetermination structures. Critically, although people selected the earlier event as the cause in the overdetermination conditions, they still imagined the recent event happening differently.

This result appears to be inconsistent with some earlier work finding that people are more inclined to imagine the counterfactual to earlier events in overdetermination structures (Walsh \& Byrne, 2004: Experiment 3). So, it is important for us to consider why this tension arises. Importantly, we do not think that this is a failure to replicate because other researchers have found similar results using overdetermination scenarios (Mandel, 2003). We offer three potential explanations. One explanation is that this disparity has to do with the different dependent measures of counterfactual thinking. As we mention above, in Walsh and Byrne's studies (and in others, e.g., Kominsky \& Phillips, 2019), the researchers asked participants to identify the counterfactual that would have made a difference to the outcome. We did not ask participants to identify the counterfactual that would have made a difference to the outcome in the scenario. Instead, our measure focused on the imagined counterfactual alternative and avoids potential conflation with the causal question. We are unsure if participants in Walsh and Byrne's study conflate the counterfactual question with the causal question, but this is one potential explanation for the disparity in the findings. Future work will have to determine the extent to which this potential conflation occurs with measures used in previous work. 
RECENCY EFFECTS IN CAUSAL JUDGMENT

A second explanation for this apparent disparity relates to participants' tendency to blame. In Walsh and Byrne's scenario, the players lost, and participants blamed the first player more frequently than the second (2004). In turn, participants might have been motivated to identify the first player in response to the counterfactual measure because they wanted to blame him. In our scenarios, by contrast, there were no losing outcomes, so no blame should have been attributed to the agents. Thus, our participants would not have been motivated to identify the counterfactual alternative to the first agent by their desire to blame him. This difference in potential blame attributions could explain the differences in counterfactual considerations. Importantly, we note that if this cognitive process affected counterfactual judgments in Walsh and Byrne's studies, it might not be a purely motivational bias; rather, because people tend to consider the counterfactuals to blameworthy or norm-violating actions, blame - in addition to recency — could have affected participants counterfactual considerations (See Byrne, 2016). Moreover, we note that there is some evidence against this explanation for the differences between the results: researchers have found and manipulated recency effects for counterfactual thinking in similar ways using situations with winning outcomes (Byrne et al., 2000).

A third explanation for this disparity is that the salience of counterfactual alternatives varies between the studies. While people tend to simulate the counterfactual to recent events, the context, structure, and other experimental interventions can affect the salience of counterfactual alternatives (Byrne et al., 2000; Walsh \& Byrne, 2004). Walsh and Byrne argue that the recency effect in counterfactual thinking occurs because people think about the events that produce the outcome (i.e., the winning conditions), and when the first event matches those conditions, it anchors people's consideration of the alternative possibilities, making them more inclined to consider counterfactuals to later events. In their studies, they reverse the effect when the first event does not match the winning conditions because, they argue, the first event does not anchor people's counterfactual considerations. Notably, when the first event matched the winning conditions in inclusive-disjunction cases, they found a recency effect rather than a reversal (2004: Experiment 2). Our results, in turn, are consistent with this work on counterfactual thinking. Louie making his shot first matches the winning conditions, and this fact anchors people's 
RECENCY EFFECTS IN CAUSAL JUDGMENT

counterfactual considerations, so we expect a recency effect for counterfactual thinking. As such, the general recency effect might vary by context more than previously thought. Future work should continue to investigate the potential for manipulating the salience of counterfactual alternatives and the effects that such manipulations would have on causal judgments.

Regardless of what brings about these potentially divergent findings, participants' causal judgments and imagined counterfactuals in Experiment 2 are perfectly in line with what the extended necessity-sufficiency model predicts: when people show the recency and primacy effect for causal judgment, they show a recency effect for counterfactual thinking. While we have not shown that people spontaneously simulate these counterfactual alternatives, our forced-choice binary measure of which counterfactual alternatives people are more likely to consider indicates that there is overall a recency effect for simulating counterfactual alternatives. These results justify a further, direct investigation into whether people's imaginings of particular counterfactuals actually affect their causal judgments in the way that we have suggested. As such, in Experiment 3 we manipulate which counterfactuals people imagine and investigate if these manipulations affect their causal judgments in the ways predicted by the extended necessity-sufficiency model.

\section{$4 \quad$ Experiment 3}

The aim of this experiment was to manipulate people's counterfactual thinking and to investigate whether it affected their causal judgments in the ways predicted by the extended necessity-sufficiency model. In this experiment, we had participants read one version of a vignette where we manipulated recency and causal structure just as we did in previous experiments. Using a paradigm inspired by previous work on normality and causal judgments (Kominsky \& Phillips, 2019; Phillips et al., 2015), we then had participants either imagine the early event happening differently (early-counterfactual-manipulation conditions), imagine the recent event happening differently (late-counterfactual-manipulation conditions), or describe the events that occurred in the passage (control) before measuring their agreement with the causal statement just as we did in previous experiments. 
RECENCY EFFECTS IN CAUSAL JUDGMENT

For the extended necessity-sufficiency model, we had four specific predictions. First, in the control conditions, we predicted an interaction between recency and structure, reflecting the recency and primacy effects and replicating the results of Experiments 1 and 2.

Second, in the early-counterfactual-manipulation conditions, relative to the control, we predicted a weaker interaction (or no interaction) between recency and causal structure. On the extended necessitysufficiency model, if people are encouraged to simulate the counterfactual to the early event—against their natural tendency — they should show a weaker interaction between recency and structure. In jointcausation conditions, people should be more inclined to judge the early event as causal, because they would be encouraged to simulate the counterfactual alternative to it and these simulations highlight that the early event made a difference to the outcome. This change in causal strength in the joint-causation conditions should weaken the recency effect. In overdetermination cases, people should be less inclined to judge the early event as causal, because they would be encouraged to simulate the counterfactual alternative to it and these simulations highlight that the early event did not make a difference to the outcome. This change in causal strength in the overdetermination conditions should weaken the primacy effect.

Third, in the late-counterfactual-manipulation conditions, relative to the control, we predicted a stronger interaction between recency and structure. On the extended necessity-sufficiency model, if people are encouraged to consider the counterfactual to the late event-amplifying their natural tendency - they should show a slightly stronger interaction between recency and structure. In jointcausation conditions, people should be more inclined to judge the recent event as causal, because they would be encouraged to simulate the counterfactual alternative to it and these simulations highlight that the recent event made a difference to the outcome. This change in causal strength in the joint-causation conditions should strengthen the recency effect. In overdetermination cases, people should be less inclined to judge the recent event as causal, because they would be encouraged to simulate the counterfactual alternative to it and these simulations highlight that the recent event did not make a 
RECENCY EFFECTS IN CAUSAL JUDGMENT

difference to the outcome. This change in causal strength in the overdetermination conditions should strengthen the primacy effect.

Finally, we predicted that this difference in two-way interactions between the three counterfactual-manipulation conditions would manifest as a three-way interaction between recency, causal structure, and counterfactual manipulation.

\subsection{Sample size}

We aimed to recruit 156 participants per condition just as we did in Experiments 1 and 2 for a total of 1915 participants.

\subsection{Participants}

All participants were United States nationals, were born in and resided in the United States, spoke English as their first language, had a 99\% approval rating on Prolific, and had not taken part in our pilot study, Experiment 1, or Experiment 2. A total of 1923 participants completed the experiment that was programmed in Qualtrics. 43 participants reported not paying attention, so they were excluded. We excluded an additional 21 participants either who completely disregarded the task or who had taken the experiment multiple times (detailed list: https://osf.io/2zfg6/). We analyzed data from the remaining 1859 participants $\left(M_{a g e}=35, S D=13.1\right.$, Range $\mathrm{age}_{\text {age }}=[18-80], 49 \%$ female $)$. After completing the survey, participants were compensated $\$ 0.33$.

\subsection{Materials and procedure}

We advertised the experiment in the exact same way as in Experiment 1 and 2. Participants were told that they would be compensated $\$ 0.33$ upon completion of the study. Before participants entered the experiment, they read a consent form.

After consenting to participate, participants were randomly assigned to 1 of 12 conditions in a 2 (Recency: early, late) $\times 2$ (Structure: joint causation, overdetermination) x 3 (Counterfactual Manipulation: early-counterfactual manipulation, control, late-counterfactual manipulation) betweenparticipants design. Each participant read one of the vignettes used in Experiment 2 (Table 2). On the following page, participants were presented with instructions for the counterfactual manipulation task. 
RECENCY EFFECTS IN CAUSAL JUDGMENT

They were either asked to imagine the early event not occurring at all (early-counterfactual-manipulation condition), the late event not occurring at all (late-counterfactual-manipulation condition), or to describe what actually happened (control) (Table 3). On the following page, participants responded to the same causal question as in Experiment 2 (Table 2). After responding to the causal question, participants were asked for basic demographic information and to respond to the same attention check used in Experiments 1 and 2.

\begin{tabular}{|l|l|l}
\hline $\begin{array}{l}\text { (A) Now we would like you to imagine Louie } \\
\text { not making a 3-point shot at all. }\end{array}$ & $\begin{array}{l}(B) \text { Now we would like you to consider what } \\
\text { happened in the passage. } \\
\text { Please describe what you imagine. }\end{array}$ & $\begin{array}{l}(C) \text { Now we would like you to imagine } \\
\text { Claire not making a 3-point shot at all. } \\
\text { the passage you read. } \\
\text { (Please write at least two sentences.) }\end{array}$ \\
(Please write at least two sentences.)
\end{tabular}

Table 3. Instructions for the counterfactual manipulation used in Experiment 3. (A) Instructions for the early-counterfactual-manipulation condition in the early conditions and for the late-counterfactualmanipulation condition in the late conditions. $(B)$ Instructions for the control conditions. $(C)$ Instructions for the early-counterfactual-manipulation condition in the late conditions and for the late-counterfactualmanipulation condition in the early conditions.

\subsection{Results}

For the causal question, there was a three-way interaction between causal structure, recency, and counterfactual manipulation $\left(F(7,1847)=16.82, p<.001, \eta^{2}{ }_{p}=.06, C I[.04, .08]\right)$. There was also an effect of causal structure $\left(F(1,1847)=5.04, p=.02, \eta_{p}^{2}=.00, C I[.00, .01]\right)$ and recency $(F(1,1847)=$ $\left.39.79, p<.001, \eta_{p}^{2}=.02, C I[.01, .03]\right)$, but there was no main effect of counterfactual manipulation $\left(F(2,1847)=2.94, p=.05, \eta^{2}{ }_{p}=.00, C I[.00, .01]\right)$. Critically, the interaction between recency and structure was weaker in the early-counterfactual-manipulation conditions than in the control conditions $(b$ $=21.15, S E=7.86, t=2.68, p=.007, C I[5.72,36.58])$, but the interaction between recency and structure was not significantly stronger in the late-counterfactual-manipulation conditions relative to the control conditions $(b=-3.29, S E=7.87, t=-.41, p=.67, C I[-18.74,12.15])$. 
RECENCY EFFECTS IN CAUSAL JUDGMENT

To decompose the three-way interaction effect between structure, recency, and counterfactual manipulation, we computed separate tests of the interaction effects and then the simple main effects for each counterfactual-manipulation condition. First, we investigated the interaction of structure and recency in the control conditions. In the control conditions, there was an interaction between causal structure and recency $\left(F(1,618)=48.93, p<.001, \eta^{2}{ }_{p}=.07, C I[.04, .11]\right)$, no effect of causal structure $(F(1,618)=.27$, $\left.p=.59, \eta_{p}^{2}=.00, C I[.00, .01]\right)$, but an effect of recency $\left(F(1,618)=13.85, p<.001, \eta^{2}{ }_{p}=.02, C I[.01\right.$, .04]). We further decomposed this interaction by investigating the simple main effects for each causal structure. In line with the recency effect, participants in the joint-causation conditions agreed that the recent event $(M=13.73, S D=31.68, n=153)$ was more causal than the earlier one $(M=4.65, S D=$ $36.32, n=156)(t(307)=-2.34, p=.01, d=-.26, C I[-.49,-.04])$. In line with the primacy effect, participants in the overdetermination conditions agreed that the earlier event $(M=24.91, S D=30.95, n=$ 160) was more causal than the recent one $(M=-4.26, S D=37.07, n=153)(t(311)=7.57, p<.001, d=$ $.85, C I[.62,1.08])$ (Figure 3).

Next, we investigated the interaction of structure and recency in the early counterfactualmanipulation conditions. In the early counterfactual-manipulation conditions, there was an interaction between causal structure and recency $\left(F(1,616)=9.95, p=.001, \eta^{2}{ }_{p}=.02, C I[.00, .04]\right)$. There was an effect of causal structure $\left(F(1,616)=5.72, p=.01, \eta^{2}=.00, C I[.00, .03]\right)$ and an effect of recency $\left(F(1,616)=17.84, p<.001, \eta^{2}{ }_{p}=.03, C I[.01, .05]\right)$. We further decomposed this interaction by investigating the simple main effects for each causal structure. In the joint-causation conditions, participants' agreement with the causal statement about the early $(M=11.69, S D=29.79, n=156)$ and late events $(M=8.87, S D=33.18, n=154)$ did not significantly differ $(t(308)=.78, p=.43, d=.08, C I$ $[-.13, .31])$. As such, the recency effect was weaker in the early-counterfactual-manipulation conditions than in the control conditions $(b=-11.89, S E=5.57, t=-2.13, p=.03)$. In the overdetermination conditions, however, participants agreed that the early event $(M=13.81, S D=34.79, n=152)$ was more causal than the late event $(M=-6.10, S D=36.75, n=158)(t(308)=4.95, p<.001, d=.55, C I[.33, .78])$. 
RECENCY EFFECTS IN CAUSAL JUDGMENT

There was no evidence that the primacy effect was weaker in the early-counterfactual-manipulation conditions than in the control conditions $(b=9.26, S E=5.55, t=1.66, p=.09)$.

Next, we investigated the interaction of structure and recency in the late counterfactualmanipulation conditions. In the late-counterfactual-manipulation conditions, there was an interaction between causal structure and recency $\left(F(1,613)=51.07, p<.001, \eta^{2}{ }_{p}=.08, C I[.05, .11]\right)$. There was also an effect of causal structure $\left(F(1,613)=4.16, p=.04, \eta^{2} p=.00, C I[.00, .02]\right)$ and recency $(F(1,613)=$ $\left.8.77, p=.003, \eta^{2}{ }_{p}=.01, C I[.00, .03]\right)$. We further decomposed this interaction investigating the simple main effects for each causal structure. In the joint-causation conditions, participants agreed that the recent event $(M=13.73, S D=36.65, n=160)$ was more causal than the earlier one $(M=1.97, S D=36.48, n=$ 155) $(t(313)=-2.85, p=.004, d=-.32, C I[-.54,-.09])$. There was no evidence that the recency effect was stronger in the late-counterfactual-manipulation conditions than in the control conditions $(b=2.67, S E=$ $5.55, t=.48, p=.62)$. In the overdetermination conditions, participants agreed that the earlier event $(M=$ $17.25, S D=33.40, n=147)$ was more causal than the recent event $(M=-12.53, S D=37.51, n=155)$ $(t(300)=7.27, p<.001, d=.83, C I[.61,1.06])$. There was no evidence that the primacy effect was stronger in the late-counterfactual-manipulation conditions than in the control conditions $(b=-.61, S E=$ $5.59, t=-.11, p=.91)$. 
Counterfactual Manipulation
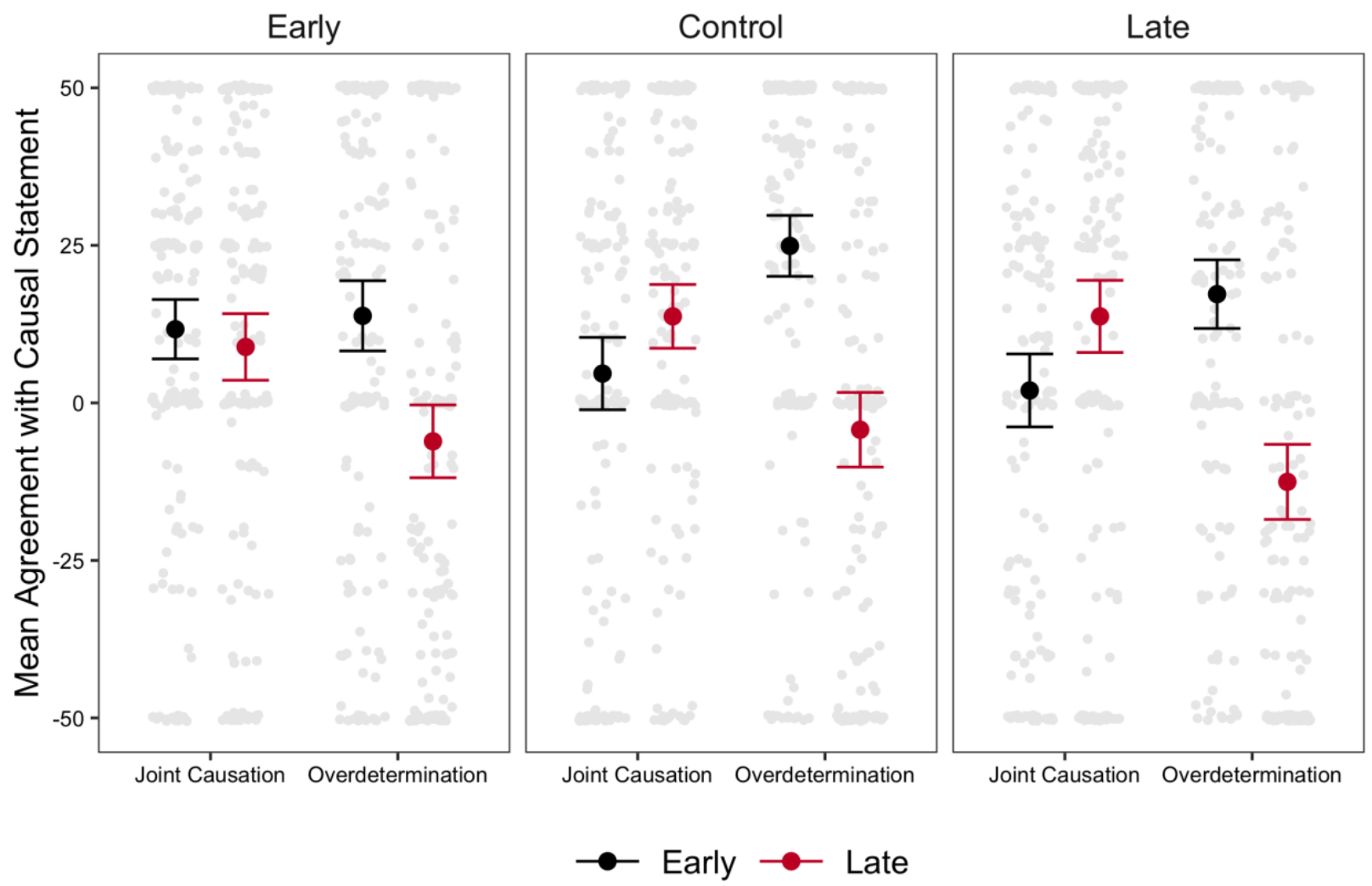

Figure 3. Mean agreement with the causal statement in Experiment 3. Error bars indicate $95 \%$ confidence intervals. Light grey points represent individual participant responses evenly jittered.

\subsection{Discussion}

These findings confirmed many of the predictions of the extended necessity-sufficiency model. In the control conditions, there was an interaction between recency and structure, reflecting the recency and primacy effects. When people were asked to imagine the counterfactual to the earlier event, we found a weaker interaction between recency and structure, no evidence for a recency effect, but also no significant reduction in the strength of the primacy effect. Moreover, when people were asked to imagine the counterfactual to the more recent event, we had no evidence for a stronger interaction between recency and structure or for stronger recency or primacy effects. 
RECENCY EFFECTS IN CAUSAL JUDGMENT

There were some unexpected results in this experiment. Interestingly, we found a qualitatively weaker interaction between recency and structure in the control conditions $\left(\eta^{2}{ }_{p}=.07, C I[.04, .11]\right)$ relative to Experiments $1\left(\eta^{2}{ }_{p}=.15, C I[.11, .19]\right)$ and $2\left(\eta^{2}{ }_{p}=.11, C I[.07, .14]\right)$. One explanation for this difference is that the instructions in our control condition encouraged people to disregard the temporal order of the events. In this condition, we had participants describe what they had read. One participant wrote " 2 friends played basketball and made a bet with some friends watching. they both made 3 point shots and won $\$ 100$ to split between them" and another wrote, "Both players made 3 point shots and won the bet." In many of the descriptions of the scenario, participants failed to describe the sequence of the events themselves; they simply stated that both events happened. While the instructions for the control condition were innocuous, the task may have encouraged participants to view each event as entirely equivalent in temporality. Participants may have anchored their responses such that early and late events were equivalent, thus they no longer engaged in the natural tendency to imagine the counterfactual to the more recent event. We resolved this concern in Experiment 5 by modifying the instructions.

Another interesting result was that the interaction between recency and structure was largely maintained in the late-counterfactual-manipulation conditions rather than strengthened as we predicted. The extended necessity-sufficiency model predicts that there should be at least a slight increase because the late-counterfactual-manipulation conditions amplify people's natural tendency to consider the counterfactual to the more recent event. We were unsure as to why this trend would occur, so we attempted to replicate it in Experiment 5.

A third interesting result was that the primacy effect seemed to be larger than the recency effect and more resistant to the counterfactual manipulation. While the recency effect was eliminated in the early-counterfactual-manipulation conditions, the primacy effect persisted. One potential explanation for the persistence of the primacy effect is that it was typically larger than the recency effect; for instance, in Experiment 2 the recency effect was smaller $(d=-.42, C I[-.64,-.20])$ than the primacy effect $(d=.93, C I$ $[.70,1.15])$. As such, the larger effect may be more difficult to manipulate, requiring more counterfactual simulations to change. We, however, have only used two different vignettes to investigate these effects, 
RECENCY EFFECTS IN CAUSAL JUDGMENT

so we cannot be confident that this trend is consistent. We aimed to see if the larger size of the primacy effect is consistent using a range of vignettes in Experiment 4.

At this point, reviewers identified an important potential confound in our experiments: normality. In our vignettes, participants read about agents making shots "right at the buzzer." Participants may have thought that it was more statistically unlikely (i.e., statistically abnormal) for people to make a buzzer shot than it was just to make some shot at the beginning of the game. So, it is possible that when we manipulated recency, we also varied the perceived statistical likelihood of each event. This potential confound is important because we know that people are more inclined to simulate counterfactuals to statistically abnormal or exceptional events (e.g., Byrne, 2016; Kahneman \& Miller, 1986). So, if participants in our experiments saw more recent events as statistically abnormal, then normality and not recency could have affected their counterfactual considerations, thus their causal judgments. If this were so, the recency and primacy effects would not result from recency—but possibly from normality. As such, if this potential confound explained the previous results, it would be possible that the findings from Experiments 1-3 would be an investigation of the normality effects originally investigated by Icard and colleagues (2017). Moreover, we know that recency and normality effects on causal judgment interact in unique ways (Reuter et al., 2014). So, this potential confound could have also affected the accuracy of the findings resulting from our manipulations.

We investigated this potential confound, and we found some support for it (See Supplemental Experiment 1). In this supplemental experiment, we ran a version of Experiment 2 where we added a question about how likely participants thought the early and the late event were to occur. The experiment replicated the recency and primacy effects for causal judgment. It also showed that people judged the early event to be more likely to occur than the late event. So, participants perceived the later event as more statistically abnormal than the early event. As such, it is possible that our previous findings could have been a result of this difference in perceived normality rather than recency.

We aimed to avoid this potential confound by developing a range of new vignettes that did not show a difference in perceived statistical normality. If we detected an interaction between recency and 
RECENCY EFFECTS IN CAUSAL JUDGMENT

causal structure in these new vignettes where the late event is not seen as more statistically abnormal, then we could be confident that recency and primacy effects arise independently of those produced by normality. We tested this prediction in Experiment 4. If this prediction was confirmed, we assumed that we could then use one of these vignettes to get more accurate results from our manipulation task without the potential interference of statistical normality (Experiment 5).

$5 \quad$ Experiment 4

There were two aims of this experiment. First, we wanted to replicate the interaction between recency and causal structure and the recency and primacy effects using multiple vignettes where the late event was not perceived as more statistically abnormal than the early one. To do so, we developed three new vignettes inspired by related work on causal judgment (Henne et al., 2019). Just as in Experiments 1 and 2, we manipulated recency and causal structure, and we measured participants' agreement with a causal statement. Second, we wanted to investigate whether people thought the late event was more statistically abnormal than the early one. To do so, after we asked participants the causal question, we asked them to identify which event they believed was more likely to occur on a continuous measure. If participants thought that the late event was more statistically abnormal than the early one, then there would be evidence that recency and primacy effects are explained by perceived differences in statistical normality. If participants did not perceive the late event as more statistically abnormal than the early one, then there would be evidence that the recency and primacy effects arise independently of normality effects. We predicted that we would find the same pattern of causal judgments as we do in Experiments 1 and 2 with multiple vignettes, and we predicted that the late event would not be perceived as more statistically abnormal than the early event.

\subsection{Preregistration}

The preregistration for this experiment is available on OSF (https://osf.io/bmrf5). This preregistration includes sample size justification, preregistered hypothesis, and planned statistical analyses.

\subsection{Participants}


RECENCY EFFECTS IN CAUSAL JUDGMENT

All participants were United States nationals, were born in and resided in the United States, spoke English as their first language, and had a 99\% approval rating on Prolific. A total of 1112 participants completed the experiment that was programmed in Qualtrics. 24 participants reported not paying attention, so they were excluded. We analyzed data from the remaining 1089 participants $\left(M_{\text {age }}=33, S D=11.6\right.$, Range $_{a g e}=$ [18-81], 50\% female). After completing the survey, participants were compensated $\$ 0.30$.

\subsection{Materials and procedure}

We advertised the experiment in the exact same way as we did in Experiments 1-3. Before participants entered the experiment, they read a consent form.

After consenting to participate, participants were randomly assigned to 1 of 12 conditions in a 2 (Recency: early, late) $\times 2$ (Structure: joint causation, overdetermination) $x 3$ (Vignette: coffee, implosion, watch) between-participants design. Each participant read one vignette (example in Table 4; see Supplementary Materials for all vignettes and dependent measures). On the following page, participants responded to a causal question on the same scale as in Experiments 1-3 (Table 4). After responding to the causal question, participants were presented with the same vignette again on a new page. Below the vignette, participants were asked "In the story that you just read, which event do you believe was more likely to happen?" They responded on a -50-50 slider scale where the numeric value was not displayed: $50=[$ Early Event $], 0=$ Both were equally likely, $50=[$ Late Event $]$. For instance, for the early conditions in the implosion vignette, participants responded on the following scale: $-50=$ Tom switching on knob A, $0=$ Both were equally likely, $50=$ Tom switching on lever D. Participants were then asked for basic demographic information and to respond to the same attention check used in Experiments 1-3. 
RECENCY EFFECTS IN CAUSAL JUDGMENT

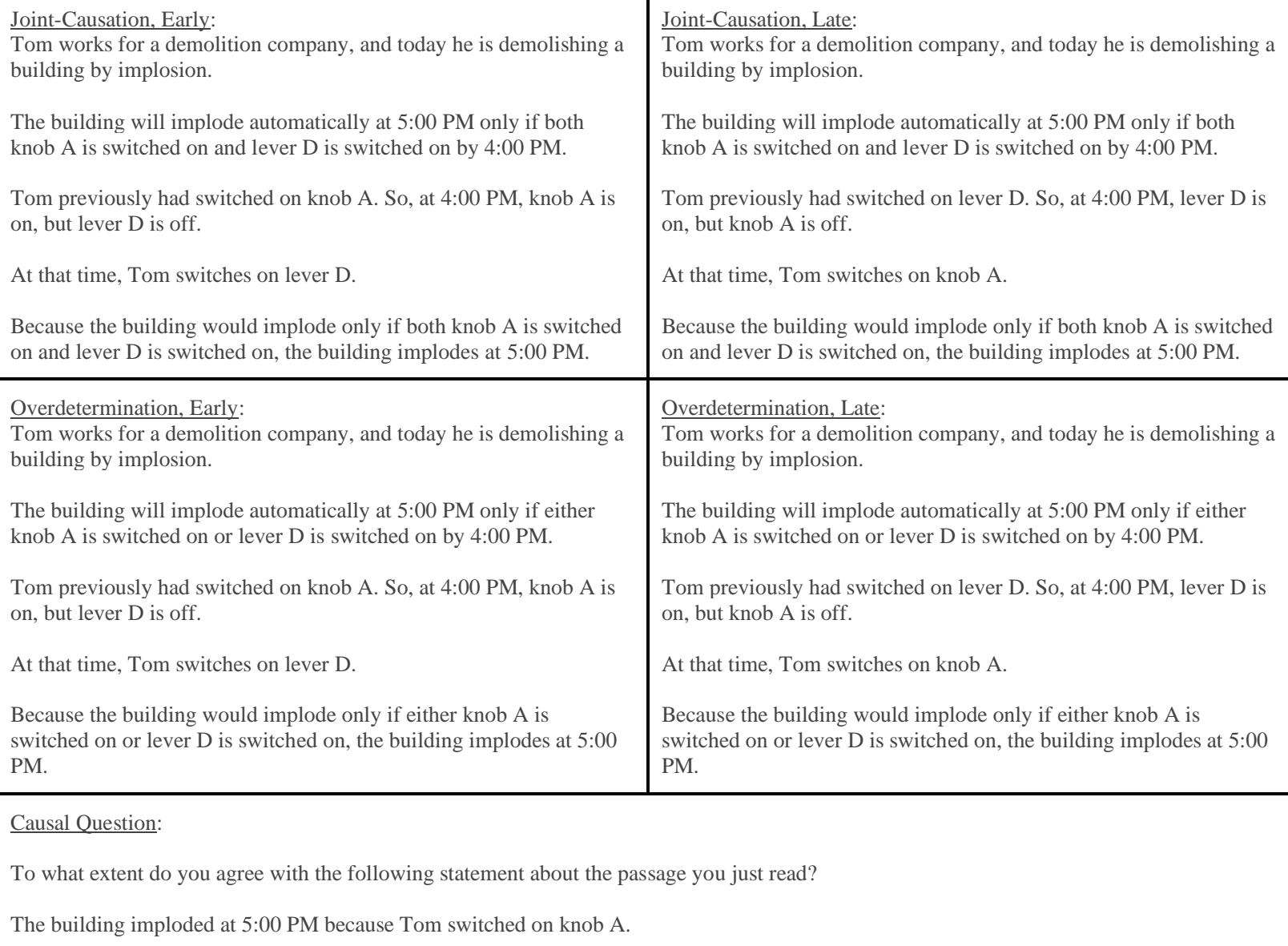

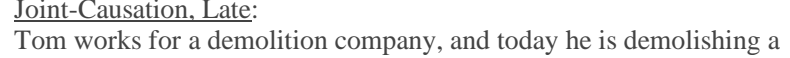 \\ building by implosion. \\ The building will implode automatically at 5:00 PM only if both \\ Tom previously had switched on lever D. So, at 4:00 PM, lever D is \\ on, but knob A is off. \\ Because the building would implode only if both knob A is switched \\ Overdetermination, Late: \\ Tom works for a demolition company, and today he is demolishing a \\ The building will implode automatically at 5:00 PM only if either \\ knob $\mathrm{A}$ is switched on or lever D is switched on by 4:00 PM. \\ previously had switched on lever D. So, at 4:00 PM, lever D is \\ At that time, Tom switches on knob A. \\ Because the building would implode only if either knob $\mathrm{A}$ is \\ switched on or lever D is switched on, the building implodes at 5:00 \\ PM. \\ on, but
}

Table 4. Implosion vignette used in Experiment 4 and the dependent variable.

\section{$5.4 \quad$ Results}

For this experiment, we fitted data to linear mixed-effects models, and we included vignette as a random intercept in the models. We assessed significance for fixed effects via Satterthwaite's degrees of freedom method. We reported the descriptive statistics in the Supplementary Materials.

For the causal question, there was an interaction between causal structure and recency $(F(1,1082)$ $\left.=115.04, p<.001, \eta_{p}^{2}=.10, C I[.07, .12]\right)$. There was no effect of causal structure $(F(1,1082)=2.70, p=$ $\left..10, \eta_{p}^{2}=.00, C I[.00, .01]\right)$ or recency $\left(F(1,1082)=1.10, p=.29, \eta_{p}^{2}=.00, C I[.00, .01]\right)$.

To decompose the interaction effect between structure and recency, we computed tests of simple main effects. In line with the recency effect, participants in the joint-causation condition agreed that the recent event $(M=31.19, S D=26, n=269)$ was more causal than the earlier one $(M=6.63, S D=35.1, n$ 
RECENCY EFFECTS IN CAUSAL JUDGMENT

$=268)\left(b=-24.6, S E=2.67, t=-9.21, p<.001, C I_{b}[-29.8,-19.3], d=-.79, C I_{d}[-1,-.58]\right)$. In line with the primacy effect, participants in the overdetermination condition agreed that the earlier event $(M=$ 25.56, $S D=33.95, n=278)$ was more causal than the recent one $(M=5.38, S D=40.82, n=273)(b=$ 20.2, $\left.S E=3.2, t=6.31, p<.001, C I_{b}[13.9,26.5], d=.53, C I_{d}[.33, .73]\right)$ (Figure 4).

For the statistical normality question, participants reported that the late event was more likely to happen than the early event $(M=4.09, S D=29.18, n=1088)(t(1087)=4.63, p<.001, d=.28, C I[.16$, .40]) (Figure S3). We also ran a preregistered exploratory analysis to investigate whether participants' judgments of normality varied as a function of recency and structure (See Supplemental Analysis 1). There was some evidence that this tendency varied as a function of recency or causal structure, but these findings were not in the direction whereby statistical normality would explain the recency and primacy effects (Figure S2). 


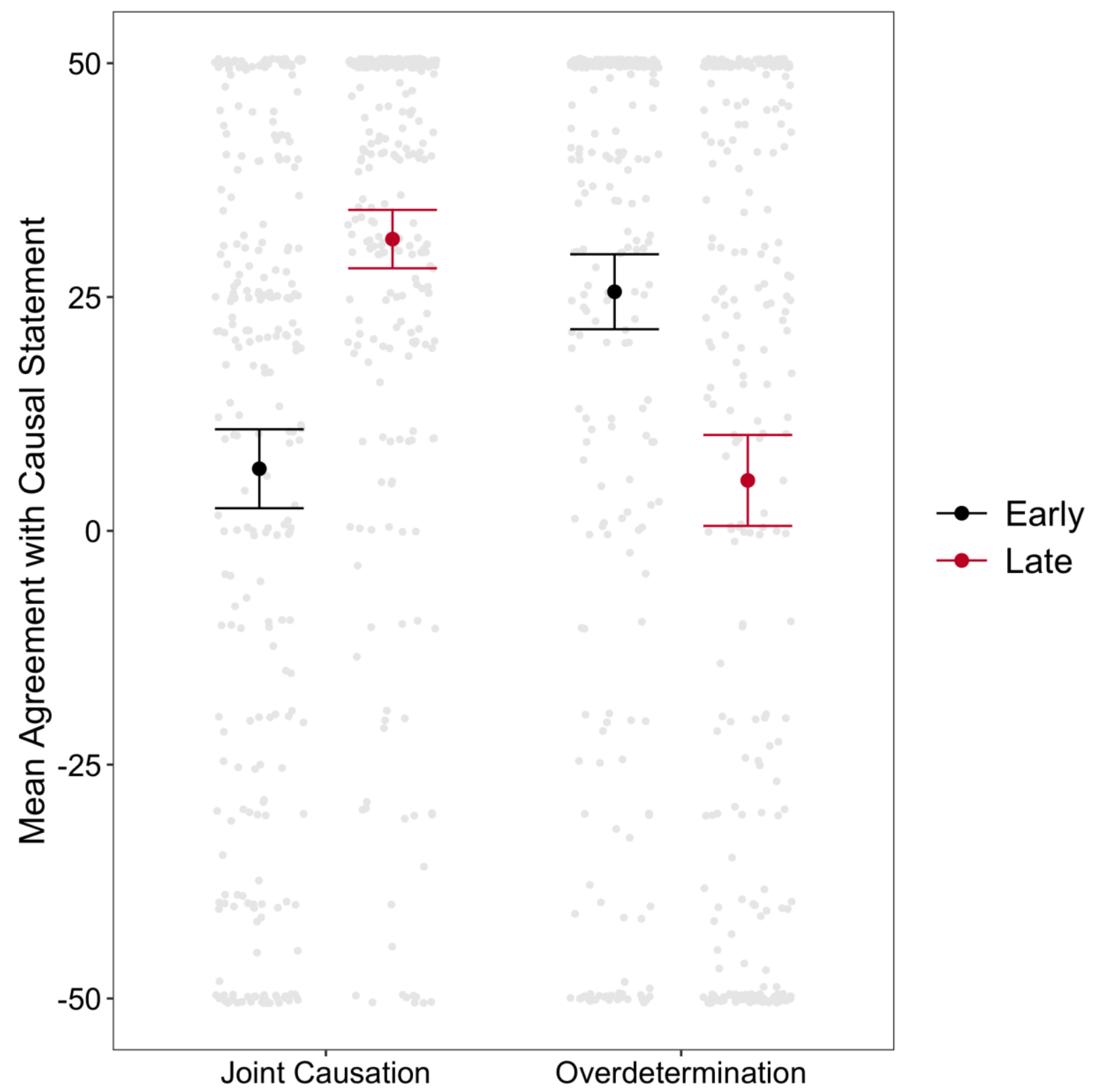

Figure 4. Mean agreement with the causal statement in Experiment 4. Error bars indicate 95\% confidence intervals. Light grey points represent individual participant responses evenly jittered.

\subsection{Discussion}

In Experiment 4, we replicated the interaction between recency and causal structure, reflecting the recency and primacy effects for causal judgment, with three new vignettes. These results confirm the robustness of these effects. Participants also thought that the late event was more likely to happen than the 
RECENCY EFFECTS IN CAUSAL JUDGMENT

early event; that is, it is not the case that participants thought that the recent event was more statistically abnormal than the earlier one. This finding is actually in the opposite direction of what we would expect if normality explained the recency and primacy effects that we found in previous experiments. Taken together, it is clear that these effects arise from temporal differences between the events and not from the perceived statistical normality of the events.

These results also help inform some of the findings from Experiment 1-3 wherein the primacy effect tended to be much larger than the recency effect (see discussion in Section 4.4). Interestingly, when we replicated the effects with a wider range of vignettes in this experiment, the effect size of the primacy effect $\left(d=.53, C I_{d}[.33, .73]\right)$ was qualitatively smaller than that of the recency effect $\left(d=-.79, C I_{d}[-1,-\right.$ .58]). Because of this variation, we are not confident in any consistent comparative difference in the effect sizes between the recency and primacy effects. To get an accurate estimate of the effect sizes, researchers should conduct a meta-analysis and investigate these potential differences across a range of experiments.

In this experiment, we found that the recency and primacy effects arise independently from related effects of perceived normality on counterfactual thinking and causal judgments. But we found some evidence suggesting that perceived statistical normality is a potential confound in Experiments 1-3 (Supplemental Experiment 1). So, while it seemed unlikely that normality completely explained these results, it was possible that normality explained the results from our previous experiments or that normality and recency interacted in these experiments. Critically, this possibility undermined some inferences from the results of Experiment 3. As such, we needed to run an improved version of Experiment 3 using a vignette that did not show a difference in perceived statistical normality such that the recent event was more statistically abnormal than the early event. We did this in Experiment 5. For this improved version of Experiment 3, we used the implosion vignette (Table 4). For this vignette, participants judged the late event as being slightly more statistically normal than the early event $(M=$ $3.65, S D=27.71, n=366)(t(365)=2.52, p=.01, d=.26, C I[.06, .47])$, but importantly we had no evidence that this tendency varied as a function of recency and structure $(p=.59)$.

$6 \quad$ Experiment 5 
RECENCY EFFECTS IN CAUSAL JUDGMENT

This experiment aimed to replicate the results from Experiment 3 while avoiding the potential confound of perceived statistical normality. To do so, we used the implosion vignette from Experiment 4. Just as in previous experiments, we manipulated recency and causal structure, and we measured participants' agreement with a causal statement. Just as in Experiment 3, we had participants read a single vignette (Table 4), and then we had them either imagine the early event happening differently (earlycounterfactual-manipulation conditions), imagine the recent event happening differently (latecounterfactual-manipulation conditions), or describe the events that occurred in the passage (control) before indicating their agreement with the causal statement. We also modified our materials such that the control condition emphasized the temporal order of events (see discussion in Section 4.4). We had the same predictions as we did in Experiment 3 (see Section 4), and we preregistered our hypotheses and exploratory analyses.

\subsection{Preregistration}

The preregistration for this experiment is available on OSF (https://osf.io/8xhks). This preregistration includes sample size justification, preregistered hypothesis, and planned statistical analyses.

\subsection{Participants}

All participants were United States nationals, were born in and resided in the United States, spoke English as their first language, had a $99 \%$ approval rating on Prolific, and had not participated in Experiment 4. A total of 1332 participants completed the experiment that was programmed in Qualtrics. 35 participants reported not paying attention, so they were excluded. We excluded an additional 13 participants who either completely disregarded the task or wrote completely irrelevant material (detailed list: https://osf.io/64czp/). We analyzed data from the remaining 1284 participants $\left(M_{\text {age }}=34, S D=11.9\right.$, Range $_{\text {age }}=[18-78], 51 \%$ female). After completing the survey, participants were compensated $\$ 0.35$. 6.3 Materials and procedure

We advertised the experiment in the exact same way as we did in Experiments 1-4. Before participants entered the experiment, they read a consent form. 
RECENCY EFFECTS IN CAUSAL JUDGMENT

After consenting to participate, participants were randomly assigned to 1 of 12 conditions in a 2 (Recency: early, late) $\times 2$ (Structure: joint causation, overdetermination) $\times 3$ (Counterfactual Manipulation: early-counterfactual manipulation, control, late-counterfactual manipulation) betweenparticipants design. Each participant read one version of the implosion vignette used in Experiment 4 (Table 4). On the following page, we presented participants with instructions for the counterfactual manipulation task. We either asked them to imagine the early event not occurring at all (earlycounterfactual-manipulation condition), the late event not occurring at all (late-counterfactualmanipulation condition), or to describe what actually happened in the passage (control) (Table 5). On the following page, participants answered the same causal questions on the same scale as in Experiment 4 (Table 4). After responding to the causal question, participants were asked for basic demographic information and to respond to the same attention check used in Experiments 1-4.

\begin{tabular}{|c|c|c|}
\hline $\begin{array}{l}\text { (A) Now we would like you to imagine that } \\
\text { Tom had not switched on knob A at all. }\end{array}$ & $\begin{array}{l}\text { (B) Now we would like you to consider what } \\
\text { happened in the passage you just read. }\end{array}$ & $\begin{array}{l}\text { (C) Now we would like you to imagine that } \\
\text { Tom had not switched on lever D at all. }\end{array}$ \\
\hline $\begin{array}{l}\text { Please describe what you imagine. } \\
\text { (Please write at least two or three sentences.) }\end{array}$ & $\begin{array}{l}\text { Please describe what actually happened in } \\
\text { the passage and the order in which the events } \\
\text { occurred. } \\
\text { (Please write at least two or three sentences.) }\end{array}$ & $\begin{array}{l}\text { Please describe what you imagine. } \\
\text { (Please write at least two or three sentences.) }\end{array}$ \\
\hline
\end{tabular}

Table 5. Instructions for the counterfactual manipulation used in Experiment 5. (A) Instructions for the early-counterfactual-manipulation condition in the early conditions and for the late-counterfactualmanipulation condition in the late conditions. $(B)$ Instructions for the control conditions. $(C)$ Instructions for the early-counterfactual-manipulation condition in the late conditions and for the late-counterfactualmanipulation condition in the early conditions.

\subsection{Results}

For the causal question, there was a three-way interaction between causal structure, recency, and counterfactual manipulation $\left(F(7,1272)=25.33, p<.001, \eta^{2}{ }_{p}=.12, C I[.09, .15]\right)$. There was also a main effect of causal structure $\left(F(1,1272)=4.18, p=.04, \eta^{2}{ }_{p}=.00, C I[.00, .01]\right)$, recency $(F(1,1272)=6.16, p$ 
RECENCY EFFECTS IN CAUSAL JUDGMENT

$\left.=.01, \eta_{p}^{2}=.00, C I[.00, .01]\right)$, and counterfactual manipulation $\left(F(2,1272)=3.70, p=.02, \eta^{2}=.01, C I\right.$

$[.00, .01])$. Critically, the interaction between recency and structure was weaker in the early-

counterfactual-manipulation conditions than in the control conditions $(b=31.82, S E=9.62, t=3.30, p<$

$.001, C I[12.95,50.69])$, and the interaction between recency and structure was stronger in the late-

counterfactual-manipulation conditions than in the control conditions $(b=-31.61, S E=9.55, t=-3.30, p$

$<.001, C I[-50.37,-12.86])$.

To decompose the three-way interaction effect between structure, recency, and counterfactual manipulation, we computed separate tests of the interaction effects and then the simple main effects for each counterfactual-manipulation condition. First, we investigated the interaction of structure and recency in the control conditions. In the control conditions, there was an interaction between causal structure and recency $\left(F(1,405)=38.44, p<.001, \eta^{2} p=.09, C I[.05, .13]\right)$. There was also a main effect of causal structure $\left(F(1,405)=4.63, p=.03, \eta^{2} p=.01, C I[.00, .03]\right)$, but there was not a main effect of recency $\left(F(1,405)=.13, p=.71, \eta_{p}^{2}=.00, C I[.00, .01]\right)$. We further decomposed this interaction by investigating the simple main effects for each causal structure. In line with the recency effect, participants in the jointcausation conditions agreed that the recent event $(M=28.15, S D=29.53, n=107)$ was more causal than the earlier one $(M=5.73, S D=36.31, n=100)(t(205)=-4.88, p<.001, d=-.68, C I[-.96,-.39])$. In line with the primacy effect, participants in the overdetermination conditions agreed that the earlier event $(M$ $=20.32, S D=37.31, n=99)$ was more causal than the recent one $(M=-.20, S D=36.64, n=103)(t(200)$ $=3.94, p<.001, d=.55, C I[.27, .83])($ Figure 5).

Next, we investigated the interaction effect of structure and recency in the early-counterfactualmanipulation conditions. In the early-counterfactual-manipulation conditions, there was no interaction between causal structure and recency $\left(F(1,428)=2.71, p=.10, \eta^{2}{ }_{p}=.01, C I[.00, .02]\right)$. There was also no main effect of causal structure $\left(F(1,428)=.21, p=.64, \eta^{2} p=.00, C I[.00, .01]\right)$ or recency $(F(1,428)=$ $\left.3.41, p=.06, \eta^{2}{ }^{2}=.01, C I[.00, .03]\right)$. So, we found no evidence that causal judgments varied as a function of recency and causal structure in the early-counterfactual-manipulation conditions, and we were not justified in investigating the simple main effects. 
RECENCY EFFECTS IN CAUSAL JUDGMENT

Next, we investigated the interaction of structure and recency in the late-counterfactualmanipulation conditions. In the late-counterfactual-manipulation conditions, there was an interaction between causal structure and recency $\left(F(1,439)=129.93, p<.001, \eta^{2}{ }_{p}=.23, C I[.17, .28]\right)$. There was no main effect of causal structure $\left(F(1,439)=3.49, p=.06, \eta^{2}{ }_{p}=.01, C I[.00, .03]\right)$, but there was a main effect of recency $\left(F(1,439)=7.32, p=.007, \eta_{p}^{2}=.02, C I[.00, .04]\right)$. We further decomposed this interaction by investigating the simple main effects for each causal structure. In the joint-causation conditions, participants agreed that the recent event $(M=31.12, S D=30.81, n=113)$ was more causal than the earlier one $(M=2.66, S D=37.65, n=108)(t(219)=-6.16, p<.001, d=-.82, C I[-1.11,-.55])$. There was no evidence that the recency effect was stronger in the late-counterfactual-manipulation conditions than in the control conditions $(b=6.03, S E=6.74, t=.89, p=.37)$. In the overdetermination conditions, participants agreed that the earlier event $(M=34.26, S D=27.91, n=111)$ was more causal than the recent event $(M=-11.85, S D=40.03, n=111)(t(220)=9.95, p<.001, d=1.34, C I[1.06$, 1.61]). The primacy effect was stronger in the late-counterfactual-manipulation conditions than in the control conditions $(b=-25.59, S E=6.78, t=-3.77, p<.001)$. 
Counterfactual Manipulation
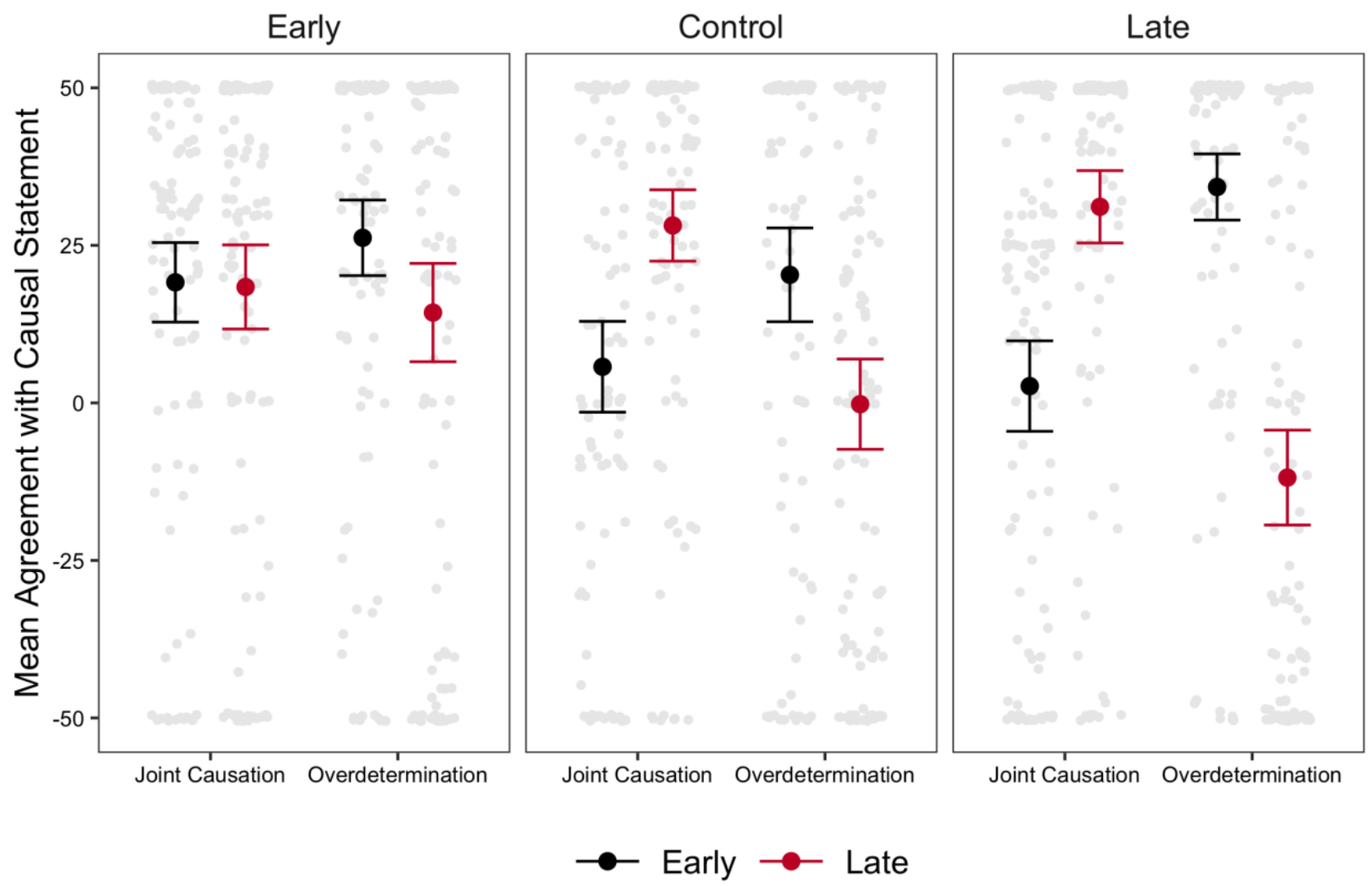

Figure 5. Mean agreement with the causal statement in Experiment 5. Error bars indicate 95\% confidence intervals. Light grey points represent individual participant responses evenly jittered.

\subsection{Discussion}

The results of Experiment 5 confirmed the predictions of the extended necessity-sufficiency model. There was a three-way interaction between recency, structure, and counterfactual manipulation. Critically, the interaction between recency and structure was weaker in the early-counterfactual-manipulation conditions and stronger in the late-counterfactual-manipulation conditions relative to the control conditions. In the control conditions, there was an interaction between recency and structure, replicating the recency and primacy effects. When we encouraged people to imagine the counterfactual to the early event, we found no evidence of an interaction between recency and structure. Moreover, when we encouraged people to imagine the counterfactual to the recent event, we saw a stronger interaction between recency and 
RECENCY EFFECTS IN CAUSAL JUDGMENT

structure. Because of these results and the results of Experiment 4, we can be confident that these patterns of judgments did not result from differences in perceived statistical normality but rather solely from the effect of temporal differences on counterfactual thinking and the relationship between counterfactual thinking and causal judgments. These results yield strong support for extending the necessity-sufficiency model to recency.

As we mentioned in Experiment 3 (section 4.4), the primacy effect again seemed to be more resistant to the counterfactual manipulation than the recency effect (Figure 5). On the extended necessitysufficiency model, we expected that when we encouraged people to imagine the counterfactual to the earlier event, we would see a weaker interaction between recency and structure or no interaction. In Experiment 3, we found a weaker interaction but only a qualitatively weaker primacy effect. In Experiment 5, however, finding no interaction, we were not justified in investigating the primacy effect by looking at the simple main effects. To investigate the persistence of the primacy effect in the earlycounterfactual-manipulation conditions, we performed unplanned post-hoc pairwise comparisons (Tukey HSD corrected) that were not preregistered. There was no evidence of a primacy effect in the earlycounterfactual-manipulation conditions $(t(1272)=2.47, p=.35, d=34, C I[.07,61])$. As such, we have no evidence that the primacy effect is more persistent than the recency effect. We suspect that the trend in line with a primacy effect is due to the fact that the counterfactual manipulation affects only the extent to which people see the events as sufficient in overdetermination conditions, while it affects the extent to which people see the events as both necessary and jointly sufficient in joint-causation conditions.

\section{$7 \quad$ General Discussion}

In Experiments 1-5, we found an interaction between recency and causal structure, reflecting the recency effect in joint-causation structures and the primacy effect in overdetermination structures. In Experiment 2, we found that people tend to consider counterfactual alternatives for more recent events regardless of causal structure. In Experiment 3 and 5, we found that manipulating which counterfactual people consider (early or late) affected their causal judgments in the ways predicted by the extended necessity-sufficiency model. Specifically, encouraging people to consider the counterfactual to the early event weakened the 
RECENCY EFFECTS IN CAUSAL JUDGMENT

interaction between recency and structure, while encouraging people to consider the counterfactual to the late event strengthened the interaction between recency and structure. In sum, we found strong support for the extended necessity-sufficiency model of causal judgment.

Our results are consistent with previous work on causal judgments. Earlier work showed that people tend to judge the more recent event as causal, relative to earlier events, in joint-causal structures (Lagnado \& Channon, 2008; Reuter et al., 2014; Spellman, 1997). Other work showed that people tend to judge the earlier event as causal, relative to recent events, in overdetermination structures (Chang, 2009; Lombrozo, 2010; Walsh \& Sloman, 2011). Not only did we conceptually replicate both of these effects, but we have also unified these distinct lines of inquiry. We used a continuous measure in order to investigate the strength of the causal judgments (Icard et al., 2017), and we used new scenarios in order to directly investigate the interaction between causal structure and temporal location. Our findings are robust (Experiment 4), and we provide a unified explanation for them with the strong support of our experimental manipulations (Experiments 3 and 5).

At the beginning of this article, we suggested that the extension of the necessity-sufficiency model, a computation model of causal strength, to recency effects on causal judgment could explain both of these effects. At this point, it might be helpful to some readers to consider some of the details of the original model. Uniquely, the necessity-sufficiency model weights the extent to which the causal factor is necessary for the outcome (necessity strength) by the probability of considering the counterfactual and the extent to which it is sufficient for the outcome (sufficiency strength) by the probability of considering what actually happened (for details, see Icard et al., 2017). As such, the more that people are inclined to imagine a counterfactual to the potential cause, the greater the weight of the necessity strength, and the more that people are inclined to think of the potential cause occurring just as it did, the greater the weight of the sufficiency strength. On this model, causal strength, or the degree to which a factor is regarded as the cause of an outcome, is equal to the weighted sum of the necessity strength and the sufficiency strength of the potential causal factor. This model's assumptions allow it to predict different patterns of causal judgments for different causal structures. In joint-causation structures, it predicts that people will 
RECENCY EFFECTS IN CAUSAL JUDGMENT

be more inclined to judge an event as causal when they are more inclined to simulate the counterfactual alternative to it because of an increase in the weighting of the necessity strength. In overdetermination cases, however, people will be less inclined to judge an event as causal when they are more inclined to simulate the counterfactual alternative to it because of a decrease in the weighting of the sufficiency strength.

Our proposed extension of this model adds a novel, critical assumption: people are more inclined to simulate counterfactuals to more recent events. This extension allows us to explain different patterns of causal judgments about more recent or earlier events in different causal structures. In joint-causation structures, people are more inclined to judge the recent event as causal (the recency effect), because they simulate the counterfactual alternative to it and these simulations highlight that the recent event made a difference to the outcome (i.e., there is an increase in the weighting of the necessity strength). In overdetermination cases, people are less inclined to judge the recent event as causal (the primacy effect), because they simulate the counterfactual alternative to it and these simulations highlight that the recent event did not make a difference to the outcome (i.e., there is an decrease in the weighting of the sufficiency strength). In our experiments, we confirm that people tend to imagine the counterfactual to the recent event regardless of causal structure (Experiment 2), and our manipulation experiments (Experiment 3 and 5) lend strong support for this extension of the model.

At this point, some readers may wonder if some alternative models of causal judgment explain the recency and primacy effects and the result of our studies. Spellman's crediting-causality model (CCM) is one prominent account that might do just that (Spellman, 1997). This model holds that the causal strength of some event varies with the extent to which that event happening changes the subjective probability of the outcome happening. To see how this model predicts the recency and primacy effects, consider our original example. In overdetermination cases, Claire making her shot at the beginning of the game changes the probability of winning the bet from some undefined degree of certainty to absolute certainty. So, people judge the early event as the cause of the outcome because it greatly increases the subjective probability of the outcome occurring. The CCM, thus, correctly predicts a primacy effect. In 
RECENCY EFFECTS IN CAUSAL JUDGMENT

the joint-causation conditions, the CCM explanation is slightly different. Claire makes her shot at the beginning of the game, and people have a moderate degree of belief that Claire and Louie will win the bet if Louie makes the shot at some point. Late in the game then, people's belief that Claire and Louie will win the bet might be very low. As a result, when Louie makes the buzzer shot, participants' belief changes dramatically from low to absolute certainty. As such, the later event produces a greater change in the subjective probability of the outcome occurring than the earlier event. Thus, the CCM correctly predicts a recency effect.

While the CCM predicts the recency and primacy effect, our results in Experiment 5 are inconsistent with it. When we asked participants in Experiment 5 to imagine the counterfactual to the earlier event, we saw an elimination of the interaction between recency and structure. When we had participants imagine the more recent event happening differently, we saw the opposite effect: it strengthened the interaction. These results are predicted by the necessity-sufficiency model but not by the $\mathrm{CCM}$. The CCM predicts that the recency and primacy effects are due to changes in the subjective degree in belief that the outcome will happen, but our counterfactual manipulations aimed to affect participants' counterfactual imaginings and not to change their degree of belief that the outcome will happen. As such, our findings are consistent with work showing that the CCM fails to explain the effects of context manipulations that make certain counterfactuals salient without changing the subjective probability of the outcome (Byrne et al., 2000). There may be charitable ways to revise the CCM such that it explains our results, but we think that these revisions would be ultimately unsuccessful (see Supplemental Discussion).

Rather, these results are promising for the necessity-sufficiency model of causal judgment (Icard et al., 2017). This model was intended to explain many effects produced by the abnormal-normal distinction's impact on causal judgment, and it has made some novel predictions that are confirmed in recent work (Gerstenberg \& Icard, 2020; Icard et al., 2017; Kirfel \& Lagnado, 2018; Morris et al., 2019). Because the model assumes that people are more likely to simulate some counterfactuals than others, its general approach has been extended to explain other distinctions that affect counterfactual thinking in this way. As we mentioned in the introduction, it has been extended to explain the effect of action-inaction 
RECENCY EFFECTS IN CAUSAL JUDGMENT

differences on causal judgment (Henne et al, 2019). Our experiments suggest that the model can also explain these recency effects on causal judgment—although it may also explain other domains of counterfactual thinking like control (see Byrne, 2016).

Critically, our new extension of the necessity-sufficiency model also offers a novel solution to a puzzle that counterfactual theories and models of causation have been wrestling with for decades: it offers a counterfactual explanation for judgments of late preemption (i.e., the primacy effect). Late preemption judgments are tricky for counterfactual theories because people judge that the earlier event caused the outcome despite the fact that the outcome still would have happened in imagined counterfactuals where the earlier event had not. As such, a counterfactual account seems insufficient, and some identify this tension as detrimental. Notably, this difficulty for counterfactual accounts to explain the primacy effect has led theorists to emend counterfactual theories (Paul, 1998a; Lewis, 2000) and to modify structural equation frameworks of causation (Halpern \& Pearl, 2005; Hitchcock, 2001). This is also a motivation for adopting some kind of causal pluralism where there is a generative, or productive, concept of causation in addition to the counterfactual one (Hall, 2004; Lombrozo, 2010). On these views, what accounts for judgments of late preemption is the perception of force interacting between the cause and the effect - the early event happening physically generates the outcome - rather than anything about the consideration of counterfactuals. Our new extension of the necessity-sufficiency model, however, suggests that these kinds of changes are not necessary, for it provides a novel counterfactual explanation for judgments of late preemption. As such, counterfactual theorists and modelers do not have to look to generative theories of causal judgment and reasoning or to pluralism to account for these late-preemption judgments; there is now a counterfactual explanation that is consistent across many domains of counterfactual thinking. 8 Conclusion

In this paper, we found ample support for the recency and primacy effect for causal judgement. Using a manipulation paradigm, we also found strong support for the extended necessity-sufficiency model of causal strength, a computational counterfactual model of causal judgment. These findings should inform work on causal judgment and alternative models for causal judgment (e.g., Khemlani et al., 2014; 
RECENCY EFFECTS IN CAUSAL JUDGMENT

Quillien, 2020), and they should be extended to work on confidence in causal judgment (O'Neill et al., 2021), to moral judgment (Gerstenberg \& Lagnado, 2012), and to experimental jurisprudence (Knobe \& Shapiro, 2020).

\section{References}

Bennett, J. (2003). A philosophical guide to conditionals. Oxford University Press.

Bernstein, S. (2014). Omissions as possibilities. Philosophical Studies, 167(1), 1-23. doi:10.1007/s11098013-0229-0

Bernstein, S. (2016): Overdetermination Underdetermined. Erkenntnis 81(1), pp. 17-40, doi:10.1007/ s10670-015-9726-1

Byrne, R. M. J. (2007). The rational imagination: How people create alternatives to reality. MIT Press.

Byrne, R. M. J. (2016). Counterfactual Thought. Annual Review of Psychology, 67(1), 135-157. doi: 10.1146/annurev-psych-122414-033249

Byrne, R. M., \& McEleney, A. (2000). Counterfactual thinking about actions and failures to act. Journal of Experimental Psychology: Learning, Memory, and Cognition, 26(5), 1318-1331. doi:10.1037/0278-7393.26.5.1318

Byrne, R. M., Segura, S., Culhane, R., Tasso, A., \& Berrocal, P. (2000). The temporality effect in counterfactual thinking about what might have been. Memory \& Cognition, 28(2), 264-281. doi:10.3758/bf03213805

Chang, W. (2009). Connecting counterfactual and physical causation. Proceedings of the Annual Meeting of the Cognitive Science Society, 31(31), 1983-1987.

Fazelpour, S. (2020). Norms in counterfactual selection. Philosophy and Phenomenological Research.

Ganeri, J., Noordhof, P., \& Ramachandran, M. (1996). Counterfactuals and preemptive causation. Analysis, 56(4), 219-225. 
RECENCY EFFECTS IN CAUSAL JUDGMENT

Gerstenberg, T., Goodman, N. D., Lagnado, D., \& Tenenbaum, J. (in press). A counterfactual simulation model of causal judgment. Psychological Review.

Gerstenberg, T., Lagnado, D.A. (2012) When contributions make a difference: Explaining order effects in responsibility attribution. Psychon Bull Rev 19, 729-736. https://doi.org/10.3758/s13423-0120256-4

Gerstenberg, T., \& Icard, T. (2020). Expectations affect physical causation judgments. Journal of Experimental Psychology: General, 149(3), 599-607. https://doi.org/10.1037/xge0000670

Gerstenberg, T., Peterson, M. F., Goodman, N. D., Lagnado, D. A., \& Tenenbaum, J. B. (2017). Eyetracking causality. Psychological Science, 28(12), 1731-1744.

Goodman, N. (1947). The problem of counterfactual conditionals. The Journal of Philosophy, 44(5), 113128.

Hall, N. (2004). Two concepts of causation. Causation and Counterfactuals. MIT Press. pp. 225-276.

Halpern, J. Y., \& Hitchcock, C. (2014). Graded causation and defaults. The British Journal for the Philosophy of Science, 66(2), 413-457.

Halpern, J. Y., \& Pearl, J. (2005). Causes and explanations: A structural-model approach. Part I: Causes. The British journal for the philosophy of science, 56(4), 843-887.

Henne, P., Niemi, L., Pinillos, A., Brigard, F. D., \& Knobe, J. (2019). A counterfactual explanation for the action effect in causal judgement. Cognition, 190, 157-164. https://doi.org/10.1016/j.cognition.2019.05.006

Henne, P., O’Neill, K., Bello, P., Khemlani, S., \& Brigard, F. D. (2021). Norms Affect Prospective Causal Judgments. Cognitive Science. doi:10.31219/osf.io/2nwb4

Henne, P., Pinillos, Á., \& Brigard, F. D. (2017). Cause by Omission and Norm: Not Watering Plants. Australasian Journal of Philosophy, 95(2), 270-283. doi:10.1080/00048402.2016.1182567

Hitchcock, C. (2001). The intransitivity of causation revealed in equations and graphs. The Journal of Philosophy, 98(6), 273-299.

Hitchcock, C., \& Knobe, J. (2009). Cause and Norm. Journal of Philosophy, 106(11), 587-612. doi:10.5840/jphil20091061128

Icard, T. F., Kominsky, J. F., \& Knobe, J. (2017). Normality and actual causal strength. Cognition, 161, 80-93. https://doi.org/10.1016/j.cognition.2017.01.010

Kahneman, D., \& Miller, D. T. (1986). Norm theory: Comparing reality to its alternatives. Psychological Review, 93(2), 136-153. doi:10.1037/0033-295x.93.2.136 
RECENCY EFFECTS IN CAUSAL JUDGMENT

Kahneman, D., \& Tversky, A. (1982). The simulation heuristic. In D. Kahneman \& A. Tversky (Eds.), Judgment under uncertainty: Heuristics and biases. 201-208. New York: Cambridge University Press.

Khemlani, S. S., Barbey, A. K., \& Johnson-Laird, P. N. (2014). Causal reasoning with mental models. Frontiers in Human Neuroscience, 8, 849. https://doi.org/10.3389/fnhum.2014.00849

Kirfel, L., \& Lagnado, D. (2019). I know what you did last summer (and how often). Epistemic states and statistical normality in causal judgements. https://doi.org/10.17605/osf.io/zhvsb

Knobe, J., \& Shapiro, S. J. (2020) Proximate cause explained: An essay in experimental jurisprudence. University of Chicago Law Review. http://dx.doi.org/10.2139/ssrn.3544982

Kominsky, J. F., \& Phillips, J. (2019). Immoral professors and malfunctioning tools: Counterfactual relevance accounts explain the effect of norm violations on causal selection. Cognitive Science, 43(1). doi:10.1111/cogs.12792

Kominsky, J., Phillips, J., Gerstenberg, T., Lagnado, D., \& Knobe, J. (2015). Causal Superseding. Cognition. 137. 196-209. https://doi.org/10.1016/j.cognition.2015.01.013

Lagnado, D. A., \& Channon, S. (2008). Judgments of cause and blame: The effects of intentionality and foreseeability. Cognition, 108(3), 754-770. doi:10.1016/j.cognition.2008.06.009

Lewis, D. (1974). Causation. The Journal of Philosophy, 70(17), 556-567.

Lewis, D. (1986). Philosophical Papers, Volume II. Oxford: Oxford University Press.

Lewis, D. (2000). “Causation as Influence”, Journal of Philosophy, 97(4), 182-97. doi: 10.2307/2678389

Lombrozo, T. (2010). Causal-explanatory pluralism: How intentions, functions, and mechanisms influence causal ascriptions. Cognitive Psychology, 61(4), 303-332.

Mackie, J. L. (1974). The cement of the universe: A study of causation. Oxford: Clarendon Press.

Mandel, D. R. (2003). Judgment dissociation theory: An analysis of differences in causal, counterfactual and covariational reasoning. Journal of Experimental Psychology: General, 132(3), 419-434. https://doi.org/10.1037/0096-3445.132.3.419

McCloy, R., \& Byrne, R. M. (2000). Counterfactual thinking about controllable events. Memory \& Cognition 28, 1071-1078. https://doi.org/10.3758/BF03209355

McCloy, R., \& Byrne, R. M. (2002). Semifactual “even if” thinking. Thinking \& Reasoning, 8(1), 41-67.

Meehan, J. E., \& Byrne, R. M. J. (2005). The temporal order effect in children's counterfactual thinking. In B.G. Bara, L. Barsalou \& M. Bucciarelli (Eds.), Proceedings of the 27th Annual Conference of the Cognitive Science Society (pp. 1467-1473). Erlbaum, NJ: Cognitive Science Society. 
RECENCY EFFECTS IN CAUSAL JUDGMENT

Menzies, P. (2004). "Difference-Making in Context", in Collins, J. D., Paul, L. A, Hall, E. J. Causation and Counterfactuals, (pp. 139-180). MIT Press. doi:10.7551/mitpress/1752.003.0007

Miller, D. T., \& Gunasegaram, S. (1990). Temporal order and the perceived mutability of events: Implications for blame assignment. Journal of Personality and Social Psychology, 59(6), 11111118. doi:10.1037/0022-3514.59.6.1111

Morris, A., Phillips, J. S., Gerstenberg, T., \& Cushman, F. A. (2019). Quantitative causal selection patterns in token causation. PLoS ONE, 14(8). https://doi.org/10.31234/osf.io/upv8t

O'Neill, K., Henne, P., Bello, P., Pearson, J., \& De Brigard, F. (2021). Degrading Causation. https://doi.org/10.31219/osf.io/m5w9c

Parkinson, M., \& Byrne, R. M. (2017). Counterfactual and semi-factual thoughts in moral judgements about failed attempts to harm. Thinking \& Reasoning, 23(4), 409-448.

Paul, L. (1998a). Keeping Track of the Time: Emending the Counterfactual Analysis of Causation. Analysis, 58(3), 191-198. www.jstor.org/stable/3328689

Paul, L. (1998b). Problems with Late Preemption. Analysis, 58, 48-53. doi:10.1111/1467-8284.00101

Paul, L. A., \& Hall, E. J. (2013). Causation: A user's guide. Oxford: Oxford University Press.

Phillips, J., Luguri, J. B., \& Knobe, J. (2015). Unifying morality's influence on non-moral judgments: The relevance of alternative possibilities. Cognition, 145, 30-42.

Quillien, T. (2000). When do we think that X caused Y? Cognition. Advance Online Publication. https://doi.org/10.1016/j.cognition.2020.104410

Reuter, K., Kirfel, L., Riel, R. V., \& Barlassina, L. (2014). The good, the bad, and the timely: How temporal order and moral judgment influence causal selection. Frontiers in Psychology, 5. doi:10.3389/fpsyg.2014.01336

Samland, J., Josephs, M., Waldmann, M. R., \& Rakoczy, H. (2016). The role of prescriptive norms and knowledge in children's and adults' causal selection. Journal of experimental psychology. General, 145(2), 125-130. https://doi.org/10.1037/xge0000138

Segura, S., Fernandez-Berrocal, P., \& Byrne, R. M. (2002). Temporal and causal order effects in thinking about what might have been. The Quarterly Journal of Experimental Psychology Section A, 55(4), 1295-1305. doi:10.1080/02724980244000125

Sherman, S. J., \& McConnell, A. R. (1996). The role of counterfactual thinking in reasoning. Applied Cognitive Psychology, 10(1) 113-124.

Spellman, B. A. (1997). Crediting causality. Journal of Experimental Psychology: General, 126(4), 323348. doi:10.1037/0096-3445.126.4.323 
RECENCY EFFECTS IN CAUSAL JUDGMENT

Walsh, C. R., \& Byrne, R. M. (2004). Counterfactual thinking: The temporal order effect. Memory \& Cognition, 32(3), 369-378. doi:10.3758/bf03195831

Walsh, C. R., \& Sloman, S. A. (2011), The meaning of cause and prevent: The role of causal mechanism. Mind \& Language, 26, 21-52. doi:10.1111/j.1468-0017.2010.01409.x

Willemsen, P., \& Kirfel, L. (2018). Recent empirical work on the relationship between causal judgements and norms. Philosophy Compass, 14(1). doi:10.1111/phc3.12562 